\title{
XIX Canadian Congress of Neurological Sciences Abstracts of the Scientific Program
}

\author{
Platform Presentations
}

1.

Pet Studies of Circulation, Energy Metabolism and Acid-Base Status after Acute Cerebral Infarction in Man

A.M. HAKIM, R. POKRUPA, S. KITAMURA, A. EVANS, M. DIKSIC, Y.L. YAMAMOTO and W. FEINDEL (Montreal, Quebec)

We have so far studied by positron emission tomography (PET) a small number of patients within 48 hours of the onset of a cerebrovascular accident (CVA). The patients all suffered completed infarctions in the territory of the middle cerebral artery. They underwent clinical and CT examination prior to PET studies. Then in sequence using $\mathrm{C}^{15} 0_{2}$, ${ }^{11} \mathrm{C} 0$, and ${ }^{15} \mathrm{O}_{2}$ inhalation, regional cerebral blood flow (CBF), blood volume ( $\mathrm{CBV}$ ), metabolic rate for oxygen $\left(\mathrm{CMRO}_{2}\right)$ and oxygen extraction fractions (OEF) were assessed. Infusions of ${ }^{18} \mathrm{~F}$-fluorodeox yglucose and "C-dimethyloxazoli-dinedione ("C-DMO) were made to determine regional cerebral glucose metabolism $\left(\mathrm{CMR}_{\mathrm{glu}}\right)$ and $\mathrm{pH}$ respectively.

Clinical assessments were made at intervals up to one month after the infarction. Early CT scans were normal or showed vague hypodensities. Follow-up unenhanced scans clearly demonstrated variable regions of infarction.

Our PET data, despite some heterogeneity within the affected areas, permits the identification of two patient subgroups: in group I the ischemic region shows: decreased; $\mathrm{CBF}$ (below $10 \mathrm{mls} / 100 \mathrm{~g} / \mathrm{min}$ ), $\mathrm{CMRO}_{2}$ less than $60 \mu \mathrm{moles} / 100 \mathrm{~g} / \mathrm{min}$, and $\mathrm{CMR}_{\mathrm{glu}}$ (below $10 \mu \mathrm{moles} /$ $100 \mathrm{~g} / \mathrm{min}$ ); increased OEF (greater than 0.6 ) along with decreased "C-DMO uptake suggesting acidosis. In group II the ischemic region is characterized by relatively increased $\mathrm{CBF}$ (greater than $25 \mathrm{mls} / 100 \mathrm{~g} / \mathrm{min}$ ) and $\mathrm{CBV}$, decreased $\mathrm{CMRO}_{2}$ and $\mathrm{OEF}$ (less than 0.3 ), relatively preserved $C M R_{\text {glu }}$ and no acidosis. Patients in group I tended to have larger infarcts and more evidence of edema.

These studies are preliminary to a description of the temporal evolution of human cerebral infarction and the evaluation of different medical and surgical therapies proposed in this setting.

2.

Steroid Therapy in Ischemic Stroke

V. C. HACHINSKI and J. W. NORRIS (London, Ontario) (Toronto, Ontario)

Few controversies in the treatment of acute cerebral infarction can be more contentious than the use of corticosteroid drugs. Early enthusiasm, fanned by anecdotal reports, soon gave way to scepticism once more critical methodology was applied. Nevertheless, steroid therapy is still widely used in all forms of completed stroke, and a final verdict is awaited.

We evaluated the effects of high dose therapy in 113 consecutive patients with acute cerebral infarction admitted to an acute stroke unit with a double-blind, randomised, controlled trial using a dose of $480 \mathrm{mg}$ dexamethasone over 12 days. Diagnosis was confirmed by autopsy in
26 patients, by isotope scanning alone in 14 , and by CT scanning alone in 73. Severity of neurological deficits was measured using the Toronto stroke scoring system. Exclusion criteria comprised patients with known embolic sources, lesions older than 48 hours, and patients with mild neurological deficits, diabetes and infection.

The 59 patients receiving active drug matched closely with the 54 receiving placebo, including age. initial stroke score, delay in therapy and other variables likely to affect the result. Statistical comparisons included paired t-tests, chi.-squared analysis, and log-rank and KaplanMeier survival curves. The two groups did not differ in death rate or quality of survivorship in the acute stage.

It is increasingly clear that steroids have no significant clinical therapeutic effect in completed ischemic stroke and it is doubtful that further evaluation by larger multi-centre trial to demonstrate marginal benefits is justified.

3.

Results of extracranial-intracranial arterial bypass for intracranial internal carotid artery stenosis: review of 105 cases.

\section{N L. CHATER, R. RODRIGUEZ y BAENA and P. R. WEINSTEIN (San Francisco, California)}

Extracranial-intracranial arterial bypass was performed for intracranial internal carotid artery stenosis in 105 patients who had ischemic symptoms 1 to 3 months before operation. The degree of stenosis, measured angiographically, was $97 \%$. Surgical mortality and permanent surgical morbidity rates were each $1 \%$. During a mean follow-up period of 54 months, 22 patients died; 10 deaths were caused by cardiac disease and three were related to stroke, two of which were ipsilateral to the bypass. Seventy-three of the 82 survivors $(89 \%)$ had no further TIAs after surgery. Seven patients had a late stroke: five were ipsilateral, one was contralateral, and one was vertebrobasilar. The overall late death rate was $4 \%$ per year and the late death rate from neurologic causes was $0.6 \%$ per year. The late stroke rate was $1.5 \%$ per year and the rate of ipsilateral late stroke in patients who had a patent bypass was $0.63 \%$ per year. We conclude that extracranial-intracranial arterial bypass for symptomatic intracranial internal carotid artery stenosis is a reasonably safe and technically satisfactory procedure.

\section{4.}

Extracranial-intracranial arterial bypass for middle cerebral artery stenosis and occlusion: a review of 65 cases.

N. L. CHATER, B. T. ANDREWS and P. R. WEINSTEIN (San Francisco, California)

Forty-seven patients with middle cerebral artery stenosis (MCS) and I8 patients with middle cerebral artery occlusion (MCO) underwent extracranial-intracranial arterial bypass. Patients presented with a history of transient ischemic attacks (TIAs), reversible ischemic neurological deficits, TIAs after initial stroke, stroke-in-evolution, or completed 
stroke. The MCS ranged from $70 \%$ to $98 \%$ angiographically. In the MCS group, two patients (4.2\%) had a perioperative stroke (within 30 days of operation). There was no perioperative mortality. In the MCO group, no patient had a perioperative stroke and one patient (5.5\%) died from nonneurologic disease. The average follow-up was 34 months for patients with MCS and 63.5 months for those with MCO. TIAs resolved completely in $90 \%$ of patients with MCS and $91.6 \%$ of those with MCO. No patient with MCS had a late ipsilateral stroke, although five had a contralateral or vertebrobasilar stroke. One patient with $\mathrm{MCO}$ had a late ipsilateral stroke. The overall ipsilateral stroke rate was $1.6 \%$ per year for $\mathrm{MCS}$ and $1 \%$ per year for $\mathrm{MCO}$. The late overall mortality rate for MCS was $7.9 \%$ per year and for MCO was $2.1 \%$ per year. The bypass patency rate at late follow-up was $100 \%$.

\section{5.}

\section{Platelet Aggregation within Cerebral Arteriovenous Malfor-} mations

\section{G. R. SUTHERLAND, C. G. DRAKE, S. J. PEERLESS and W. C. VEZINA (London, Ontario)}

Turbulence within cerebral arteriovenous malformations (AVMs) may lead to endothelial disruption, platelet aggregation and thrombus formation. This hypothesis would account for many of the pathological features in AVMs including intimal hyperplasia and arterial thrombosis with or without organization. To evaluate in vivo platelet aggregation in 15 patients with AVMs, we utilized a dual isotope method employing I"In labeled platelets and ${ }^{99 \mathrm{~m}} \mathrm{Tc}$ labeled red blood cells. By utilizing two isotopes blood pool $(\ln (\mathrm{D}) / \ln (\mathrm{BP}))$ was calculated.

After a $24 \mathrm{hr}$ incubation period, 5 of the 15 patients demonstrated platelet aggregation in their AVMs with a mean $\ln (\mathrm{D}) / \ln (\mathrm{BP})$ ratio of $1.68 \pm 0.21$. Three of these AVMs were available for pathological study with all demonstrating evidence of arterial thrombosis of variable age. In the remaining 10 patients, the ratio $\operatorname{In}(\mathrm{D}) / \operatorname{In}(\mathrm{BP})$ was not signifjcantly elevated (mean $=1.11 \pm 0.1 \mathrm{I}$ ) indicating the absence of active platelet aggregation during this short interval of study. Four of these AVMs were pathologically examined, 2 of which showed evidence of arterial occlusion.

As there was no significant difference in the size, site, clinical presentation or pathological findings in these patients, our technique may be imaging an episodic phenomena occurring in all AVMs. Alternatively, there may be a fundamental unrecognized pathological difference between these two groups of patients.

\section{6.}

\section{"Supergiant"' Intracranial Aneurysms}

G. MOHR, M. BOJANOWSKI, N. MARTINEZ and J. HARDY, (Montreal, Quebec)

Eight patients (4 males, 4 females) harbouring unusually large intracranial aneurysms measuring between 4 and $8 \mathrm{~cm}$ in their largest diameter have been treated at Notre-Dame Hospital during the past twenty-five years.

Five of these patients presented with "pseudo-tumoral" symptoms related to the mass effect and three patients were admitted because of acute intracranial hemorrhage (S.A.H. orintraventricular hemorrhage). However, two patients who bled had already associated signs of raised 1.C.P. Patients with aneurysmal rupture were all in Botterell-Grade $\mathrm{V}$ and died without surgical treatment.

Four patients had clearly demonstrable calcifications on plain-skull films. All aneurysms were located on the anterior circle of Willis (one para-ophthalmic internal carotid, four supraclinoid internal carotid, 2 anterior cerebral and one middle cerebral artery). Two patients had multiple aneurysms.

Four patients underwent surgical treatment consisting in simple proximal ligation of the internal carotid artery in one case, proximal carotid ligation followed by removal of the aneurysm in one case, and direct removal and clipping of the aneurysm in two cases (one of these aneurysms was discovered incidentally during surgery for "meningioma").
One patient aged 78 years died from intercurrent neoplastic disease without surgery.

The pathology of these "supergiant" aneurysms will be discussed, particularly the partial intramural thrombosis that seems to contribute to their exceedingly large growth before rupture occurs.

\section{7.}

\section{Astrocytoma: Interval Appearance of Tumor After Negative CT Scan}

\section{R. LAMOND and P. J. MULLER (Toronto, Ontario)}

The clinical presentation and CT scans of 186 consecutive patients with astrocytoma (grades 1-4) were reviewed. A small subgroup of brain tumor patients were identified who, at the time of their presentation, had negative CT scans. There were 4 males and 2 females in this group. One had an astrocytoma grade 4,3 and astrocytoma grade 3 , and 2 had astrocytoma grade 2 . Their age range was $26-63$ years. All but one presented with seizures. The relationship between duration of prescan symptoms, tumor location, pathologic grade and CT tumor size were reviewed.

Unenhanced and enhanced CT scans were obtained on a GE8000 scanner. The long tumor diameter (LTD) and maximum perpendicular diameter (MPD) of the tumor and the entire abnormal area including edema were measured in $\mathrm{mm}$ and converted to actual size on the basis of the CT minification factor.

The mean LTD and MPD of the first positive scan were 37 and $22 \mathrm{~mm}$ respectively. The tumor sizes were not significantly different from those of the 180 patients with positive scans at first presentation in spite of the short (mean 2.3 month) interval between the last negative and first positive scan.

We conclude that a negative scan in a brain tumor suspect does not exclude the presence of tumor. Since it is reasonable to assume that the tumor was present at the symptomatic onset and since the interscan interval was too short for a sufficient number of doubling times to account for the sudden appearance of tumor, we also surmise that the tumor not only increased in size during the interval between the last negative and first positive scan, but also changed its CT absorbancy characteristics and/or altered the blood/brain barrier to allow contrast penetration and thus C'T identification of the tumor. It is possible that nuclear magnetic resonance scanning will allow the early identification of these "missed" brain tumors.

\section{8.}

Magnetic Resonance Imaging in Central Nervous System Tumors

W. D. ROBERTSON, D. LI, S. FACHE, J. MAYO, D. GRAEB, R. A NUGENT, J. S. LAPOINTE, D. PATY, V. SWEENEY, G. B. THOMPSON and F. D. DURITY (Vancouver, B.C.)

Twenty-four patients with clinically suspect central nervous system tumor and CT-proven abnormalities have had magnetic resonance imaging (MRI) scans using both single and multi-slice imaging techniques. A combination of inversion-recovery and spin-echo sequences using different repetition rates, inversion times, and echo delays were obtained. The majority of cases had scans performed in both the axial and coronal plane. Lesions of the posterior fossa usually had scanning performed in the sagittal plane as well.

The computed tomographic and magnetic resonance images were both studied to determine: (1) the extent of the lesion, (2) separation of the tumor from surrounding edema, (3) assessment of mass effect, (4) specificity of diagnosis, and (5) what information is displayed by one technique that is not available on the corresponding technique.

Initial analysis of the data indicates MRI has an advantage in assessing low-grade cerebral hemisphere lesions and all lesions of the posterior fossa where coronal and sagittal planes aid in displaying the abnormal anatomy. MRI has difficulty in separating a malignant tumor from its surrounding edema. CT scans with contrast enhancement are more useful in displaying more aggressive lesions and separate the tumor from the surrounding edema. Both techniques display mass effect well.

Comparison between the two techniques will be made. 


\section{9.}

Intracellular Hydrogen Peroxide and Rate of Tumour Growth

\author{
R. F. DEL MAESTRO, W. McDONALD and E. STROUDE(London, \\ Ontario)
}

During studies carried out to assess the intracellular enzymatic scavenging mechanism available to experimental and human malignant glial tumors, a consistent increase in intracellular catalase was observed. Subcellular localization studies in intracerebral $\mathrm{C} 6$ astrocytoma grown in Wistar rats suggested that this increased catalase was predominantly localized in the cytoplasmic and mitochondrial fractions. Similar studies carried out on intraoperative samples of human glioblastoma multiforme have shown that the tumour mitochondria contain much more catalase than mitochondria obtained from normal human cortex.

Since catalase reduces hydrogen peroxide to water, we have hypothesized that the intracellular concentration of hydrogen peroxide may be important in determining the rate of tumour growth.

Aminotriazole was used to decrease intracellular catalase in the $\mathrm{C} 6$ astrocytoma cell line in vitro and produced statistical decreases in catalase at $10^{-6}-10^{-4} \mathrm{M}$ concentration after 48 hours. These concentrations were associated with an increased growth rate of the C6 astrocytoma line in vitro. Hydrogen peroxide added to the growth medium also caused accelerated growth.

Our results suggest that increased levels of intracellular hydrogen peroxide are associated with accelerated growth of C6 astrocytoma in vitro.

Supported by the Medical Research Council of Canada Grant MA-8104

\section{0.}

\section{Intraoperative Localization With Real Time Ultrasound}

\section{M.C. BOYD and P. STEINBOK (Vancouver, B.C.)}

High resolution ultrasound has been used intraoperatively on fortyfive patients with various lesions. The technique used is quickly and easily carried out under sterile conditions in the operating room. Successful localization of both primary and metastatic tumors of various sizes, depths and consistencies has been made prior to extirpation or biopsy. Several of the latter were done through small burr holes. AVMs, abcesses, bone fragments from trauma, gliotic epileptic foci and ventricles for shunt placement have been readily found. No significant complications have been encountered. There were, however, three failures. In one case the lesion was visualized with the ultrasound but the biopsy taken was not diagnostic. In two other cases the lesion was so superficial that it could not be visualized in the low frequency transducer's near field. A new technique is now used to circumvent this problem. An overall reduction in operating time and unnecessary trauma to the patient has resulted from more accurate localization of pathology intraoperatively with real time ultrasound.

11.

\section{Fluorescent Histochemical Study of RNA in Paediatric Neural} Tumours

\section{H. B. SARNAT, B. CURRY, N. B. NEWCASTLE and C. TREVENEN (Calgary, Alberta)}

Acridine orange forms fluorescent complexes upon binding with nucleic acids, RNA emitting a brilliant orange colour and DNA fluorescing yellow-green. Acridine orange has been applied to tissue sections of muscle biopsies to demonstrate denervated and regenerating muscle fibers, but it is also potentially useful in the CNS because neurons have a higher RNA content than other cells including neuroglia. In this study acridine orange was applied to nervous system tumours of 44 infants and children. Among neuronal tumours, well differentiated cells of ganglioneuroblastomas and gangliogliomas (6 cases) showed much stronger RNA fluorescence than poorly differentiated cells of neuro- blastoma ( 6 cases), medulloblastoma ( 7 cases) and retinoblastoma ( 1 case). In the glial series, astrocytomas of various grades (17 cases) showed a gradient opposite that of the neural series: the strongest RNA fluorescence was seen in the least well differentiated and most pleomorphic cells. Ependymomas ( 2 cases) and primitive neuroepitheliomas ( 2 cases) exhibited moderate cytoplasmic RNA fluorescence, while a choroid plexus papilloma was similar to normal choroid plexus. A craniopharyngioma and an Ewing sarcoma metastatic to brain displayed no orange fluorescence. Acute and chronic reactive astrocytes including gemistocytes in 4 non-tumour cases also did not exhibit RNA fluorescence. The acridine orange fluorochrome technique may be a useful adjunct in the histopathologic diagnosis of cerebral neoplasms. The opposite gradients of RNA concentration in tumour cells of the neuronal and glial series in relation to degree of maturation may have implications for the original differentiation of these cell lines. Such gradients are seen not only between different tumours, but also within different portions of the same tumour showing varying degrees of maturation or differentiation.

12.

\section{Multiple Daily Fractioned Radiation Therapy and Misonidazole in the Management of Malignant Astrocytoma}

H. G. THOMAS, K. H. SHIN, R. C. URTASUN, P. H. S. GEGGIE, D. FULTON, B. CURRY, M. CAVA, H. TANASICHUK (Calgary, Alberta) (Edmonton, Alberta)

Various attempts have been made to improve the effectiveness of radiation therapy in the treatment of cerebral malignant astrocytomas.

A trend favouring Multiple Daily Fractionated (MDF) radiation therapy over conventional fractionated $(C F)$ radiation therapy was identified in our previous study. In order to assess the effect of MDF with and without misonidazole, a province wide prospective randomized trial was initiated in January, 1981. By January, 1984143 patients with histologically verified Grade III and IV astrocytomas were randomized to $\mathrm{CF}$ ( $5800 \mathrm{cGy} / 6$ weeks/30 fractions), MDF (6140 cGy/4.5 weeks/69 fractions at 89 cGy every three hours, three times a day) and MDF in combination with misonidazole $\left(1.25 \mathrm{gm} / \mathrm{m}^{2}\right.$ three times weekly for the first three weeks). One hundred and seventeen patients were evaluated for this report (38 CF, $40 \mathrm{MDF}$, and $39 \mathrm{MDF}$ and misonidazole). The median survival time from diagnosis is 29 weeks for CF group, 57 weeks for MDF group and 54 weeks for MDF and misonidazole group.

The one year actuarial survival rate from surgery is $21 \%$ for $\mathrm{CF}$ group, $52 \%$ for MDF group and $51 \%$ for MDF and misonidazole group. The important prognostic factors are type of fractionation, Karnofsky performance status, age of patient and histologic grading of the tumor.

\section{3.}

\section{I-125 Brachytherapy for Malignant Gliomas}

\section{F. DA SILVA and P. H. GUTIN (San Francisco, California)}

Because methods used to treat malignant gliomas are not effective, new therapeutic approaches must be evaluated. Many localized systemic tumors can be cured by surgery and radiation therapy, but malignant gliomas are refractory to this approach. Doses of radiation delivered by conventional beam teletherapy that might sterilize the tumor cannot be given because of the risk of (often fatal) radiation necrosis. Because malignant gliomas are essentially localized tumors and recur at the primary site in $80 \%$ of patients, a local therapy might have advantages over conventional teletherapy. Implantation of radioactive sources directly into tumor allows high doses of radiation to be delivered locally at low dose rates with relative sparing of normal brain. The availability of computed tomography-directed stereotaxy, an afterloaded catheter implantation system, and high activity $1-125$ sources makes it possible to place sources accurately into tumors, to determine dosimetry with relative precision, and to remove sources from the tumor after the desired dose of radiation has been delivered.

We have used this approach to treat recurrent malignant gliomas (37 patients) and to provide a "boost" of local radiation immediately after 
conventional teletherapy is completed (29 patients). Patients were evaluated at two month intervals using the results of CT scans and neurologic examination. Based on these criteria, $68 \%$ of patients with recurrent tumors responded to brachytherapy (disease stabilization or tumor regression). While these results cannot be compared directly with the results of chemotherapy, brachytherapy appears to be at least as effective as the best chemotherapy regimens currently used to treat patients harboring malignant gliomas. The boost series was only recently begun, and results are not yet available. The development of focal radiation necrosis is a serious complication of the procedure; however, we have found that this is a surgically-manageable problem.

\section{4.}

The Value of Adjuvant Chemotherapy (Prednisone, CCNU and Vincristine) in the Management of Newly Diagnosed Medulloblastoma, Malignant Astrocytoma and Brainstem Glioma: Results from the Children's Cancer Study Group (CCG).

J. ALLEN, I. ERTEL, A. EVANS, D. HAMMOND, D. JENKIN and R. SPOSTO (New York, New York) (Ann Arbor, Michigan) (Philadelphia, Pennsylvania) (Los Angeles, California) (Toronto, Ontario) (Los Angeles, California)

CCG has completed three large multi-institutional randomized trials for the most common malignant primary intracranial tumors of childhood. The study design incorporated a randomization to one of two treatment groups: radiation alone ( $\mathrm{RT}$ ) and radiation plus chemotheraphy (CHT). Prior to randomization, the patients from age $2-16$ years were stratified according to criteria pertinent to each disease such as tumor size and extent of disease. CCG protocol 942 entered 232 patients with medulloblastoma. Factors associated with a favorable outcome were: localized disease at diagnosis, older age and a radiation dose to the posterior fossa $>5,000$ rads. The degree of surgical resection of the primary tumor and adjuvant $\mathrm{CHT}$ had no effect on outcome after adjustment for extent of disease and age. CCG 943 included 63 children with grade III-IV astrocytoma. Older age, the degree of resection and adjuvant chemotherapy were associated with increased disease-free survival. The median survival of the CHT group was twice as long as the control. CCG 944 randomized 69 brainstem glioma patients. The overall median survival of 11 mos. was not different from historical controls and neither surgery nor chemotherapy influenced outcome. These three studies reaffirm the importance of using contemporaneous controls in randomized trials. Certain clinical or disease parameters such as age and extent of disease may have a greater influence on survival than treatment variables. CCNU and vincristine should be included in the management of childhood malignant astrocytoma.

15.

\section{Analysis of Postoperative CT Scans in Brain Tumor Patients}

M. P. RATHBONE, J. G.CAIRNCROSS, W. H.PEXMAN, H. W. K. BARR, R. F. DELMAESTRO, R. K. COATES and D. R. MACDONALD (London, Ontario)

The length of survival in patients with malignant glioma may be directly related to the bulk of tumor removed. CT scanning is probably a more accurate way to assess the extent of tumor removal and thereby the impact of resection on survival, than the operative report. Prior to studying the influence of tumor resection on survival we have analyzed the nature and natural history of postoperative CT artifacts in brain tumor patients. Local contrast enhancement has been observed following brain surgery. Post-surgical contrast enhancement at the site of tumor resection may mimic residual enhancing tumor confounding measurements of the extent of resection. To determine when the CT scan yields the most accurate information regarding residual enhancing tumor, we performed sequential scans on ten brain tumor patients undergoing eleven operations. In six cases the tumor was resected, in five a lobectomy was performed. We found contrast enhancement along the operative margin following tumor resections but not lobectomies. Enhancement appeared as early as the fifth postoperative day, was most intense at two weeks, and persisted for several months. Prior to the fifth postoperative day areas of enhancement reflected residual tumor. Steroids did not affect the intensity, timing or duration of post-surgical enhancement. Movement and air artifacts and edema were more prominent on the first and second postoperative days than on the third and fourth. We recommend that postoperative $C T$ scans to assess residual enhancing tumor be performed on the fourth postoperative day.

16.

Intracranial Mass Lesions due to Cerebral Radiation Necrosis (Report of 3 Cases)

\section{F. B. MAROUN, J. C. JACOB, W. O. GITTENS, M. MANGAN and M. FARIDI (St. John's, Newfoundland)}

The occurrence of cerebral mass lesions following radiation therapy of intracranial neoplasm is a well known clinicopathological entity. The incidence of this delayed form of radionecrosis is difficult to estimate since this complication is rarely reported. We present three adults with radiation necrosis. Two were located in the temporal lobe and followed treatment of pituitary adenoma, and one followed radiation therapy of orbital carcinoma. Detailed clinical, radiological, and pathological data, together with a review of the literature will be presented.

17.

Hemotoporphyrin Derivative Uptake and Phototoxicity in Normal Brain Tissue

D. BOISVERT, M-K. CHENG, J. McKEAN, J. TULIP, J. CUMMINS and B. MIELKE (Edmonton, Alberta)

Clinical use of light-activated hemotoporphyrin derivative (HPD) in the treatment of malignant brain tumors is currently being investigated. We have investigated in rats $(n=103)$ the dose and time related uptake of ${ }^{3} \mathrm{H}-\mathrm{HPD}$ and the relationship between brain damage, HPD dose $(2.5-20 \mathrm{mg} / \mathrm{kg})$, and laser light $(630 \mathrm{nM})$ dose $(24-192$ Joules $)$ to the brain surface.

Brain and other tissues showed progressive increases in HPD content as the HPD dose was increased $(2-20 \mathrm{mg} / \mathrm{kg})$. Gradual decreases in all tissue HPD levels occurred between 24 and 72 hours with no further decreases between 72 and 120 hours. High levels $(3-6 \mu \mathrm{g} / \mathrm{gm})$ of HPD were measured in normal brain 24 hours following $10 \mathrm{mg} / \mathrm{kg} \mathrm{HPD}$, indicating that HPD penetrates the blood brain barrier. Considerable brain damage occurred in animals subjected to light and HPD. The damage was directly correlated with both increasing laser energy and increasing HPD dose. Cortical temperature was measured with a temperature probe during laser exposure and it was found that the temperature increase was only $1-3^{\circ} \mathrm{C}$ when cortex was exposed to laser power of $50 \mathrm{~mW}$. At laser power of $200 \mathrm{~mW}$, the cortex temperature rose by $6-18^{\circ} \mathrm{C}$, demonstrating that thermal insult was responsible for some of the damage seen at this higher energy level.

Our results indicate that damage to normal brain tissue, probably related to high tissue levels of HPD, may be a significant complication of photoradiation therapy for intracranial tumors.

18.

New Electrophysiological Methods for Study of Peripheral, Spinal, Supraspinal and Extrapyramidal Pathways

A. EISEN, M. HOIRCH and D. B. CALNE (Vancouver, British Columbia)

Previous although largely ignored observations (Upton et al., J. Neurol. Neurosurg. Psychiat. 1978, 4I: 1016) have been reexamined and extended to develop a global electrophysiological method allowing simultaneous evaluation of peripheral nerve sensory la afferent conduction and spinal and supraspinal motor and sensory pathways and spinal 
cord conduction. Additionally the method can be used to investigate certain movement disorders and complement or obviate the need for presently used more cumbersome torque motor studies. In 30 normal subjects median and peroneal nerves were stimulated using long duration stimuli to preferentially activate la afferents. Responses were recorded from the thenar and the tibialis anterior muscles on a background of moderate voluntary contraction. Somatosensory evoked potentials were simultaneously elicited using identical stimulus sites and parameters. Early (R1) and late (R2) reflex responses were induced having latencies of $27.5 \pm 1.7 \mathrm{~ms}$ and $46.2 \pm 3.4 \mathrm{~ms}$ (thenar muscle) and $30.1 \pm 1.5 \mathrm{~ms}$ and $57.8 \pm 3.1 \mathrm{~ms}$ (tibialis anterior muscle). Peripheral sensory la conduction time given by $[(\mathrm{R} /-\mathrm{M}) / 2-1]$ measured $11.3 \pm 1.0 \mathrm{~ms}$ (wrist to cord) and $11.7 \pm 1.2 \mathrm{~ms}$ (knee to cord). Central sensorimotor conduction time ( $R !-R 2) / 2$ was $9.1 \pm 1.2 \mathrm{~ms}$ (C7 root entry to cortex) and $13.9 \pm 1.3 \mathrm{~ms}$ ( $L 5$ root entry to cortex) compared to central sensory conduction times of $8.6 \pm 0.8 \mathrm{~ms}$ and $12.9 \pm 1.5 \mathrm{~ms}$ respectively. The difference of $0.9 \pm 1.6 \mathrm{~ms}$ and $1.0 \pm 1.3 \mathrm{~ms}$ for arm and leg respectively is considered to reflect a single synapse between the motor and sensory cortex and is evidence favouring the long loop transcortical hypothesis. Cord conduction time between the $\mathrm{L} 5$ and $\mathrm{C} 7$ root entries could be calculated by the formula $[(\mathrm{R} 2-\mathrm{R} 1) / 2(\mathrm{leg})-(\mathrm{R} 2-\mathrm{R} 1) / 2(\mathrm{arm})]$ and measured $4.8 \pm 1.1 \mathrm{~ms}$ giving a velocity of $96.3 \pm 5.6 \mathrm{~m} / \mathrm{sec}$. Many of these measurements have not been previously possible and their potential in disease is underway.

19.

\section{The Neural Generators of the Somatosensory Evoked Potential:} A New Hypothesis

\section{A. EISEN, K. ROBERTS and M. HOIRCH (Vancouver, British Columbia)}

Digital, unlike analogue, filtering can be designed for zero-phase shift, the filtered response remaining true to latency. Somatosensory evoked potentials (SEPs) elicited by median and peroneal nerve stimulation were recorded using bipolar cephalic montages. The averaged data was digitally filtered between $300-2500 \mathrm{~Hz}$ by applying a modified Blackman window. The median SEP showed consistent negative-positive peaks at about $16,18,19$, and $20 \mathrm{msec}$, and less constant components at 14 and $21 \mathrm{msec}$. Interpeak latencies measured $1.3 \pm 0.2 \mathrm{msec}$, reflecting a discharge frequency of $770 \mathrm{c} / \mathrm{sec}$. With peroneal nerve stimulation, components were recorded at about 24, 26, 28 and $30 \mathrm{msec}$. Their interpeak latencies averaged $1.8 \pm 0.25 \mathrm{msec}$, giving a discharge frequency of $555 \mathrm{c} / \mathrm{sec}$. The fast multispike activity was also visualized through a broader window extending over the initial $100 \mathrm{msec}$ of the SEP. It was then seen to be centered around the "N20" (arm) and the "N26" (leg) slow wave envelopes of the regular SEP. It had onset latencies in keeping with thalamic origin. Since bipolar cephalic recordings induce cancellation of most far-field potentials, the multispike activity recorded cannot simply reflect deep generators and raises important questions regarding previously accepted neural generators of the SEP. It is hypothesized that a synchronous thalamocortical discharge is responsible for the multispike activity, whereas the surrounding slow wave envelope seen using conventional recording techniques is due to a cortical generator.

20.

Topographic Visual Evoked Potential in Visually Impaired Children

P. K. H. WONG, J. JAN, K. FARRELL, S. WHITING and D. L. GREGORY (Vancouver, British Columbia)

The anatomical localization of "cortical blindness" (C.B.) in children is difficult on clinical examination. Traditional electrophysiological techniques (EEG, ERG and 1 or 2 channel visual evoked potential - VEP) have a low sensitivity in this age group. We describe an electrophysiologic method - topographic VEP mapping (VEP map) which may be useful in localizing the site of involvement.

Twenty children with a clinical diagnosis of C. B. were studied. Two hundred binocular flashes at $1 / \mathrm{sec}$ were averaged with a $512 \mathrm{~ms}$. post- stimulus epoch. Twenty-one recording channels were used, with linked mandibles as reference. Computer interpolation generated 256 maps for rapid video display.

Control children showed the direct occipital cortical component ("P100") arising mid-occipitally, which then spread symmetrically to the vertex region. The VEP maps were abnormal in all our tested children, and fell into 3 groups: (I) normal potentials at the occipital regions only, with loss or absence in the parietal and more anterior areas; (II) present but abnormal early occipital components (incl "P100"); (III) diffusely abnormal. These data suggest that C.B. in children may be associated with 3 patterns of neurophysiologic dysfunction. Pattern I may represent damage to the visual association areas, pattern II primary visual cortex, and pattern III - diffuse cerebral dysfunction.

21.

Somatosensory Evoked Potentials (SEPs) in Pediatric Cardiovascular Surgery

\section{J. TAYLOR, J. G. COLES, D. S. BORRETT and J. M. PEARCE (Toronto, Ontario)}

There is wide use of evoked potentials (EPs) to monitor sensory system function during neurosurgical and orthopedic operations in which a sensory pathway is at risk. EPs are also useful, however, in cardiovascular surgery in which the risk to the CNS is less specific but no less real. Given the limitations of clinical assessments in detecting subtle neurological changes in infants and children, we investigated the use of SEPs as objective measures of CNS function in relation to intraoperative factors which may influence CNS integrity. Pre- and postoperative SEPs alone are often not sensitive enough indicators of neurological dysfunction.

We recorded SEPs in 43 children (aged 4 mos.- 16 yrs.) undergoing repair of congenital cardiac anomalies. The children requiring intracardiac repairs underwent cardiopulmonary bypass $(\mathrm{CPB})$ with hypothermia alone $\left(\right.$ mean $21^{\circ} \mathrm{C}$ ) or with profound hypothermia (mean $11^{\circ} \mathrm{C}$ ) and complete circulatory arrest. Marked changes were seen with hypothermia; as temperatures decreased there were increases in latency, dispersion and amplitude. In the infants there were delays in the return of the cortical SEPs post-arrest, reflecting, we believe, no-reflow phenomena. A second study suggested that a high $\mathrm{pH}$ provides protective influence on the CNS during arrest. In the older children perfusion rates were manipulated and the effects of reduced blood flow on the CNS were assessed. With lower flow rates than are conventionally considered safe during hypothermic CPB the SEPs did not change and were associated with the preservation of neurologic function. The SEP changes with hypothermia and ischemia also provided critical information regarding the neural generators of the subcortical waves, allowing separation between pre-\& postsynaptic activity in the dorsal columns \& brainstem.

In these cases the SEPs provided a means of assessing (usually) subclinical damage and the function of the CNS under extreme conditions of hypothermia and ischemia. The studies were also invaluable for elucidating the generators of SEP components.

22.

Somatosensory Evoked Potentials as a Prognostic Measure in Coma in Children

R. MANGERA, M. J. TAYLOR, I. G. JAMES, J. F. EDMONDS (Toronto, Ontario) (London, England)

Recent studies investigating the prognostic utility of evoked potentials in acutely brain-injured adults have found somatosensory evoked potentials (SEPs) to be the most valuable. To pursue this in pediatrics, we studied a series of 22 children (aged .5-13 yrs, mean $7 \mathrm{yrs}$ ) admitted to the ICU with Glasgow Coma Scale (GCS) scores of 10 or less. There were 3 Reyes syndromes, 2 near-drownings, 4 hypoxic-ischemic insults, 12 head injuries and 1 intractable seizures. Daily neurological examinations were done during the ICU stay; CT scans of the head were obtained in 18. The SEPs were studied within $24 \mathrm{hrs}$ of admission, regularly over the child's stay in ICU and on follow-up at least 3 months 
later. SEPs were recorded over the cervical spine and contralateral sensory cortex in response to median nerve stimulation. Unlike the GCS and neurological exam, SEP recording was not affected by paralyzing drugs and barbiturates.

The clinical outcomes were: A) 3 brain deaths, B) 8 spastic quadraparetics, C) 4 hemiparetics and D) 7 with full neurological recovery. The spinal SEPs were normal in all cases but 3, 2 from group A, 1 from group B. The cortical SEPs were normal on day 1 and over subsequent testings in 6 patients, all from group D. No reproducible cortical SEPs on follow-up. In group C 3 children had bilaterally abnormal but asymmetrical SEPs, I had unilateral abnormalities; the abnormalities were consistent with the involved hemisphere on follow-up in all 4 patients.

In this study the initial cortical SEP recordings were highly predictive of and correlated better with eventual neurological outcome than other early assessments of the patients. Hence, we believe SEPs to be a valuable adjunct in evaluating comatose children.

\section{3.}

The Influence of Reflex Activity on Mechanical Properties of the Primate Forelimb During Imposed Displacement

\section{F. A. LENZ, Y. E. ISTL-LENZ, S. SCHLOEGEL, W. G. TATTON and R. R. TASKER (Toronto, Ontario)}

To understand how reflexes might function in the control of a whole limb, mechanical properties of the primate forelimb were computed during imposed displacement of forelimb joints. Torque motor imposed extension of the metacarpophalangeal (MCP) joint, the wrist and the elbow in naive squirrel monkeys produced reflex electromyographic (EMG) activity in the flexor muscles acting across these joints. Trials were computer sorted according to the presence or absence of an EMG reflex response and averaged results for these two groups were determined. The torque (corrected for inertial effects) and the displacement of the end of the limb segment distal to the joint were then used to estimate joint stiffness with and without a reflex response (termed "reflex" and "non-reflex", respectively).

At each joint studied, the positional dependence of torque was significantly less linear (as determined by linear regression) for non-reflex trials than for reflex trials. Since velocity-dependent (viscuous) forces were small ( $<0 \%$ of torque in reflex trials), stiffness of a joint, averaged over the trajectory, was taken as a measure of the mechanical properties of that joint. Reflex stiffness values for the three joints studied were within one standard deviation when normalized to the distance from that joint to the end of the extremity. Both the EMG reflex activity and the component of force generated by reflex activity were greater at the elbow than at the wrist and MCP joint. Therefore, reflex activity varied as if to compensate for differences in the non-reflex properties of different muscle joint systems to produce linear displacement-torque relation characterized by constant normalized stiffness. This mechanical configuration across three contiguous joints in the primate forelimb may provide a useful strategy in the control of biologic or prosthetic limbs.

Supported by T.G.H. Foundation, O.H.F., M.S.S. (Canada), M.R.C.

24.

Motor Programs for Simple Voluntary Movements in Normal Subjects and Patients with Parkinsonism or Cerebellar Dysfunction

\section{R. G. LEE, B. E. MUSTARD and D. G. WHITE (Calgary, Alberta)}

Rapid voluntary movements at a single joint are accompanied by a characteristic triphasic pattern of EMG activity from the participating agonist and antagonist muscle groups. The goal of this study was to determine whether quantitative analysis of these EMG patterns might provide information concerning the motor programs which generated the movements. Initially recordings were obtained from normal subjects trained to perform wrist flexion movements to a specified target. EMG activity from the wrist flexors and extensors was correlated with the velocity, amplitude, and force of the movement. When the amplitude and opposing load were kept constant, movements of increasing velocity were accompanied by an increase in amplitude and decrease in duration of the first agonist and the antagonist EMG bursts, and a shortening of the interburst interval. With velocity and load constant, movements of increased amplitude were accomplished by an increase in the size of the EMG bursts and a lengthening of the interburst interval.

The second part of the study involved a group of patients with either cerebellar incoordination or Parkinson's disease. In both disorders, there was a marked increase in the variability of movement strategy and associated EMG patterns during repeated single trials. Cerebellar patients showed a prolonged first agonist burst and a diminished antagonist burst, which may correlate with the tendency to overshoot a target during rapid movement. In parkinsonian patients, the maximum velocity which could be attained was reduced. The first agonist burst was often small and sometimes followed by subsequent bursts. In some cases, there was also co-activation of the antagonist.

These preliminary investigations suggest that this approach can provide useful information concerning errors in motor programming in the certain neurologic disorders.

\section{5.}

\section{Positron Emission Tomography in the Study of Disorders of the Basal Ganglia}

W. R. W. MARTIN, M. HAYDEN, D. B. CALNE, J. BECKMAN, M. BERGSTROM, R. HARROP, C. SAYRE, B. D. PATE, M. ADAMS, T. RUTH and J. ROGERS (Vancouver, British Columbia)

The role played by the basal ganglia in the control of movement remains controversial. Although disorders such as Parkinson's disease (PD) and Huntington's disease (HD) are associated with recognized abnormalities of the basal ganglia, other movement disorders such as dystonia have no known pathophysiological substrate. We have studied cerebral glucose metabolism (CMRG) using ${ }^{18} \mathrm{~F}$-fluorodeoxyglucose and positron emission tomography (PET) in patients with movement disorders and in normal subjects to assess basal ganglia function. In patients with predominantly unilateral PD $(n=9)$ the most common abnormality observed was an increase in CMRG in the lenticular nucleus contralateral to the affected limbs. Patients with early HD $(n=7)$ and normal caudate nuclei on CT scan displayed a symmetric decrease in caudate metabolism. Those with bilaterally symmetric forms of dystonia (two with Meige syndrome and one with generalized dystonia) had symmetric basal ganglia metabolism. In contrast, those with spasmodic torticollis $(n=9)$ frequently had decreased CMRG in the putamen on the side to which the head turned. These studies demonstrate the utility of PET in revealing in vivo functional abnormalities in the brain in man.

\section{6.}

\section{The Role of Somatosensory Evoked Potentials in Patients with Cerebrovascular Disease}

\section{F. GENTILI, W. M. LOUGHEED and C. CORRADO (Toronto, Ontario)}

Despite the considerable amount of experimental data suggesting that somatosensory evoked potentials may be a sensitive index of cerebral ischaemia, the role of this technique in patients with cerebrovascular disorders remains to be defined. Bilateral recordings of somatosensory evoked potentials have been carried out in 65 patients presenting with cerebrovascular disorders including TIAs (27), stroke (8), and subarachnoid haemorrhage (30). In addition to standard analysis of amplitude, wave form, and latency, central conduction time (CCT) and interhemispheric difference (IHD) have been analyzed. In 26 patients presenting with TIAs, 53\% had abnormal prolonged CCT, (values greater than 2.5 S.D. from the mean control group). Six of eight patients presenting with fixed ischaemic deficits (R.I.N.D., minor completed stroke) had prolonged CCT. Thirty patients presenting with subarachnoid haemorrhage have been studied. Of 15 patients presenting in Grade 1 or 2, 3 patients had prolonged and 12 patients normal CCT, with no patient developing pre or postoperative spasm. Of 15 patients presenting in grade 3 to 5,10 had abnormally prolonged CCT; six of 
these patients developed symptomatic vasospasm. In summary, analysis of somatosensory evoked potentials can provide an additional, non-invasive means of evaluating patients with cerebrovascular disease. Our data suggests that the SSEPs can be of both diagnostic and prognostic value. In patients with subarachnoid haemorrhage, early, persistent alteration in SSEP parameters (prolonged CCT, increased IHD) was more likely to be associated with poor neurological outcome. In patients with stroke, those with early return of, or retained cortical waves following the ischaemic event had a better outcome when compared with those with persistent absent responses. In patients with carotid occlusion, SSEP findings were not of benefit in helping to select patients for EC/IC bypass.

\section{7.}

\section{Delayed Evoked Potentials (EPs) in Ankylosing Spondylitis}

\section{N. PILLAY and T. HUNTER (Winnipeg, Manitoba)}

Visual, brainstem auditory and somatosensory EPs were studied in twenty patients fulfilling the New York criteria for the diagnosis of ankylosing spondylitis (AS). The mean age was 45 years (range 19-67 years) in seventeen males and three females. The mean duration of disease was 17 years (range 1-34 years). Six patients had a past history of iritis. Visual acuity was 20/20 in thirteen, 20/25 in five, and 20/40 in two. Neurological examination was normal in eighteen, with radiculopathy in the lower limbs in two patients. The cervical spine was involved clinically in fifteen and radiologically in twelve but none had atlantoaxial subluxation or spinal fractures.

Abnormal visual evoked potentials (VEPs) were recorded in fourteen (70\%) with P100 latency delay in thirteen and interocular latency difference of $7 \mathrm{msec}$. or greater in six patients. Somatosensory evoked potentials (SEPs) were abnormal in thirteen $(65 \%)$ with latency delays in cervical $(\mathrm{N} 13)$ /or scalp recorded $(\mathrm{N} 20)$ peaks on median nerve stimulation. Three patients had prolonged central conduction (I-V) on brainstem auditory evoked responses.

Significantly impaired VEPs or SEPs did not appear to be related to severity, duration or activity of disease. EPs are known to be of value in the early diagnosis of multiple sclerosis (MS). Our data indicates that caution should be exercised in using abnormal evoked potentials in the diagnosis of multiple sclerosis if the patient has AS. Further study of these electrophysiological abnormalities may lead to a better understanding of the pathogenesis of AS.

28.

\section{Electrophysiological Characteristics of the Acute Compartment Syndrome}

\section{VOULTERS and W. F. BROWN (London, Ontario)}

Acute increases in intracompartmental pressures and subsequent ischemic damage of the muscles and nerves within the compartment may follow trauma in the region. We have studied 4 cases of traumainduced anterior compartment syndromes using various electrophysiological techniques.

These studies have shown, in the severe cases seen 3 or more weeks after the injury, electrical silence in wide areas throughout the compartment, no visible or electrical response to direct stimulation of compartment muscles, and a total loss of excitability in the nerves which pass through the compartments. In less severely affected cases, patches of electrical silence alternate with patches of abundant fibrillation potentials in muscles within the affected compartment.

Electrical silence and the loss of any response to direct muscle stimulation are strong indicators of infarction and non-viability of muscle fibers. Fibrillation potentials, although an indication of denervation, at least indicates the presence of viable muscle fibers within the compartment, which has the potential of accepting new innervation, should regenerating motor axons be able to reach them. The presence of fibrillation potentials and enhanced insertional activity is, therefore a better prognostic sign of the potential for recovery beyond 3 weeks than persistent electrical silence in the muscle indicating as it probably does, permanent loss of the muscle fibers.

29.

\section{Saphenous Vein Patch Grafts in Carotid Endarterectomy}

\section{J. R. LITTLE and B. S. BRYERTON (Cleveland, Ohio)}

The clinical and early postoperative intravenous digital subtraction angiogram (IVDSA) findings were studied in 34 patients undergoing 40 consecutive conventional carotid endarterectomies and 36 patients undergoing 40 consecutive carotid endarterectomies with a saphenous vein patch graft (SVPG). IVDSA's were considered normal after 31/40 conventional carotid endarterectomies and $36 / 40$ carotid endarterectomies with SVPG $(p=0.13)$. The internal carotid artery $(I C A)$ origin was consistently larger after carotid endarterectomy with SVPG. Ballooning at the endarterectomy site occurred with similar frequency in both groups, that is, after 3 conventional carotid endarterectomies and 2 carotid endarterectomies with SVPG. In the conventional group. early symptomatic ICA thrombosis occurred in 1 patient and early symptomatic common carotid artery (CCA), and ICA, and external carotid artery (ECA) thrombosis occurred in I patient. Silent ICA occlusion was seen in 1 patient in the conventional group. There were no occlusions in the SVPG group (i.e.. $p=0.12$ ). Stenosis (i.e.. $\leqslant 33 \%$ at the distal end of the ICA arteriotomy was found after 2 conventional carotid endarterectomies and after 1 carotid endarterectomy with a SVPG. Stenosis (i.e., $\leqslant 33 \%$ ) at the proximal end of the CCA arteriotomy was found after I carotid endarterectomy in each group. Three patients in the conventional group suffered a postoperative cerebral infarct and one patient in the SVPG suffered a postoperative brain stem infarct. The findings of the study suggested the routine use of a SVPG in carotid endarterectomy.

\section{0.}

\section{Calcium Antagonism in Cerebral Vasospasm}

R. LEBLANC, W. FEINDEL, Y. L. YAMAMOTO, J. G. MILTON, C. P. HODGE and M. M. FROJMOVIC (Montreal, Quebec)

We have studied the effects of calcium antagonism on vasospasm of the large arteries at the base of the brain and of the smaller cortical arteries. Vasospasm was induced by the subarachnoid injection of platelet rich plasma (PRP) and $25 \mathrm{uM}$ of ADP, causing maximum aggregation. Calcium antagonism was produced by topical application of verapamil. In the transclivally exposed basilar artery of the cat the subarachnoid injection of PRP-ADP produced a mean vasoconstriction of $40.7 \%$ at 5 minutes after its injection. The topical application of verapamil, with the arteries still in spasm, produced a mean dilatation of $52.7 \%$ at 5 minutes after application. This persisted in a decremental fashion for the 60 minute period of observation by which time the previously constricted arteries had returned to their pre-spasm diameters. Through a large hemispheric craniectomy, in dogs, the effects of calcium antagonism on the epicerebral arteriovenous (AV) transit time and on epicerebral perfusion were documented by the measurement of arterial diameters, fluorescein angiography, and krypton-85 regional blood flow analysis. The subarachnoid injection of PRP-ADP produced a mean vasoconstriction of $38.2 \%$ at 10 minutes after injection and verapamil produced a mean dilatation of the constricted arteries of 19.5\% 10 minutes after its application. There was a mean acceleration of the AV transit time of 4.5 seconds after verapamil compared to the spasm value. Regional epicerebral blood flow was $60.3 \mathrm{ml} / 100 \mathrm{~g} / \mathrm{min}$ in the control state, $38.2 \mathrm{ml} / 100 \mathrm{~g} / \mathrm{min}$ during vasospasm and $85.9 \mathrm{ml} / 100$ $\mathrm{g} / \mathrm{min}$ after verapamil. The topical application of verapamil can dilate the previously constricted large arteries of the base of the brain and smaller cortical arteries. This dilatation is associated with acceleration of the AV transit time and increased epicerebral blood flow. The magnitude of these changes may reflect hyperemia and a shunting phenomenon. 
31.

\section{Prostacyclin-Thromboxane System and Cerebral Vasospasm in} Experimental Subarachnoid Hemorrhage

\author{
R. C. CHAN, F. A. DURITY and G. B. THOMPSON (Vancouver, \\ British Columbia)
}

Subarachnoid hemorrhage (SAH) was induced in 50 rabbits by injecting 1.25 c.c. $/ \mathrm{kg}$ of autologous arterial blood into the cisterna magna. The animals were evenly divided into 5 groups: control, Prostacyclin ( $\left(\mathrm{PGI}_{2}\right)$, Carbacyclin, Thromboxane $\mathrm{A}_{2}$ synthetase inhibitor OKY-1581 and Nutralipid groups. Radiographic vertebro-basilar arterial spasm was demonstrated on the third day post SAH in the control animals This decreased in the Prostacyclin and the Carbacyclin groups, and was absent in the OKY-1581 and the Nutralipid groups. Cerebral blood flow using Xenon-133 failed to reveal any significant difference between the Prostacyclin, the Carbacyclin and the control groups, but flows in the Nutralipid and the OKY-1581 groups were significantly higher. There was a good correlation between the clinical status and the cerebral blood flow. Intracytoplasmic vacuolation and detachment of the vascular endothelium seen ultrastructurally, may account for the impaired synthesis of Prostacyclin $\mathrm{PGI}_{2}$. Exogenous Prostacyclin and Carbacyclin decrease vasospasm but fail to improve the cerebral perfusion. OKY-1581 blocks the synthesis of the potent vasoconstrictor Thromboxane $A_{2}$. Nutralipid contains fatty and amino acids that inhibit platelet aggregation, and block Thromboxane $A_{2}$ production.

32.

\section{Nimodipine and Chronic Vasospasm in Monkeys}

M. NOSKO, B. WEIR, C. KRUEGER, D. COOK, S. NORRIS, T. OVERTON and D. BOISVERT (Edmonton, Alberta)

Twenty-four female cynomolgous monkeys underwent induction of a subarachnoid hemorrhage (SAH) by direct surgical placement of an autologous hematoma against the major anterior cerebral arteries in the right basal subarachnoid spaces (day 0 ). The monkeys were randomized to one of four groups and were blindly treated with one of nimodipine $\mathrm{PO}$ q8h $\times 7$ days in doses of 3,6 or $12 \mathrm{mg} / \mathrm{kg}$ or with placebo. Indices monitored before and after SAH included neurological status, angiographic cerebral vessel calibre, cerebral blood flow and CT scan. The incidence and the difference in the mean vessel calibre reduction (day 7) was not significantly different among the four groups. One animal receiving nimodipine $12 \mathrm{mg} / \mathrm{kg}$ PO q8h developed a delayed neurological deficit on day 5.

Ring preparations of the middle cerebral arteries (MCA) were prepared from three animals of each group and dose-effect curves to varying concentrations of norepinephrine, 5-hydroxytryptamine and potassium chloride were obtained. There was a significant reduction in the response of the clot side MCA relative to the non-clot side to all three agonists. The clot side contractility was not influenced by nimodipine treatment. The non-clot side MCA of the $12 \mathrm{mg} / \mathrm{kg}$ treatment group demonstrated enhanced reactivity to all three agonists.

Oral nimodipine does not prevent chronic vasospasm or delayed ischemia in the primate model tested. Oral nimodipine at the doses tested enhances reactivity of normal cerebral vessels but does not affect the reactivity of arteries in chronic spasm to the agonists tested.

\section{3.}

\section{The Carotid "Stump"'Syndrome}

B. G. BENOIT, N. A. RUSSELL, G. CANTU-REYNA and B. K. MALLYA (Ottawa, Ontario)

The relatively uncommon occurrence of ischemic symptoms arising in the territory of an occluded internal carotid artery, may have one of several etiologies. Among these, may be emboli of atheromatous debris or platelet-fibrin material, arising from a non-obliterated proximal remnant of the carotid artery - the "stump". These emboli, which could also arise from the external or distal common carotid arteries, pass to the target hemisphere or eye through anastomotic channels, particularly the ophthalmic artery.

In the interval 1979-1983, we have treated 9 patients for embolization presumed to have arisen from the carotid "stump", or adjacent vessels. The average age was 64 years, with an equal sex distribution. Seven patients presented with hemisphere T.I.A.'s, 4 with amaurosis fugax, and 2 with both. The carotid "stump" measured from 2 to $12 \mathrm{~mm}$ in depth, and there was evidence of significant external carotid artery stenosis in half the cases.

Eight cases were subjected to operation, consisting of intraluminal shunting to the external carotid artery, and excision of the internal carotid artery remnant with over-sewing of the origin. In 4 cases, an external carotid endarterectomy was also performed. There was no morbidity associated with these procedures, and in all cases the preoperative symptoms ceased entirely. The patient who declined operation, was placed on Coumadin, and continued to have spells of amaurosis fugax 3 times weekly.

Since surgical treatment is safe and effective, the "stump" syndrome should be seriously considered in all patients being evaluated for ischemic symptoms, occurring in the territory of an occluded internal carotid artery.

34.

Spontaneous Dissection of Carotid and Vertebral Arteries; Management Considerations

B. G. BENOIT, N. A. RUSSELL, J. D. GRIMES, B. LACH, J. G. D'ALTON and D. ATACK (Ottawa, Ontario)

Spontaneous dissections of the great vessels, are being reported with increasing frequency, due to wider recognition of the clinical-radiological correlations. Within the past 5 years, we have recognized 14 such cases, 2 of which occurred in the vertebral arteries. The widely held opinion, that carotid dissections result in a minor self-limiting clinical course, was borne out by an excellent recovery in 7 of our patients. They had presented with hemicranial pain, oculo-sympathetic palsy, with or without transient neurological disturbance. Treatment consisted of anticoagulation for various times, followed by repeat angiography or Doppler analysis.

However, 4 carotid cases presented initially with major cerebral infarction, 1 requiring surgical decompression, and another succumbing to distal embolization to the middle cerebral artery. Another unusual case, found subsequently to represent an Ehler's-Danlos type IV syndrome, presented with a carotid cavernous fistula and subsequently died of multiple arterial dissections.

One dissecting aneurysm of the vertebral artery, resulted in death from a massive subarachnoid hemorrhage. In another, a "flap type" vertebral dissection, caused a recurring transient lateral medullary syndrome, despite adequate anticoagulation.

The variable outcomes described in this series, serve to emphasize the need for early recognition and adequate treatment, of this potentially lethal condition.

35.

\section{Cerebral Amyloid Angiopathy}

R. LEBLANC, R. G. COSGROVE, K. MEAGHER-VILLEMURE and R. ETHIER (Montreal, Quebec)

The case records of 24 patients with amyloid angiopathy are reviewed. There were 14 males and 10 females aged 58 to 93 years (mean: 75 years). Eight patients (Group A) presented with dementia or other medical problem. Sixteen patients (Group B) presented with multicompartmental intracranial hemorrhage producing an attered level of consciousness and hemiparesis ( 8 cases) or coma and pathological posturing ( 8 cases). Group A patients had a protracted course and died of pneumonia or other medical complication. In Group B, 11 patients died of intracranial hypertension and its complications, while 4 had a protracted deterioration and died of pneumonia. Four patients were 
operated on and 1 survived. Autopsy revealed cardiac hypertrophy and/or nephrosclerosis in hypertensive patients and Alzheimer's disease in demented patients. There was amyloid deposition in the leptomeningeal and cortical arteries and arterioles in all patients and in association with the hematomas. The hematomas involved the frontal and parietal lobes in 7 cases each, the temporal lobe in five cases, the occipital lobe in 8 cases and the thalamus in 1 case. Subarachnoid hemorrhage was common. Ventricular hemorrhage occurred in four patients; and 4 patients had a subdural hematoma. CT scanning, performed in 11 patients, correlated well with the autopsy finding. In 4 Group A patients it demonstrated diffuse, non-specific cerebral atrophy. In 7 Group B patients it demonstrated hematomas involving the cortex and subcortical white matter and the subarachnoid and ventricular extensions of blood. Cerebral amyloid angiopathy is a major cause of cerebral hemorrhage in the elderly and has a poor prognosis. The unusual location of the hematomas, the cortical involvement and the subarachnoid extension of blood, seen on CT scanning, are useful in differentiating amyloid angiopathy from other causes of intracranial hemorrhage in this age group.

36.

\section{Myasthenia Gravis in the Elderly}

D. T. CRISP, P. ST. GEORGE-HYSLOP and J. G. HUMPHREY (Toronto, Ontario)

40 patients over 60 years of age with myasthenia gravis (MG) without thymoma have been studied. Immunologic and physiologic parameters, response and complications of therapy and prognosis are reviewed. 30 patients were male and 7 had ocular findings only. 20 of $22(91 \%)$ had elevated antiacetylcholine receptor $(\mathrm{AChR})$ antibody titres. Single fibre EMG studies showed abnormal jitter in 12 of $14(86 \%)$ and neuromuscular blocking in 6 of $14(43 \%)$. The correlation coefficient between $\mathrm{AChR}$ levels and jitter was $0.71(\mathrm{p}<.01)$.

36 patients $(90 \%)$ were treated with prednisone. The average maximum dosage was $70 \mathrm{mg}$ on alternate days with a range from $30 \mathrm{mg}$ alternate days to $100 \mathrm{mg}$ per day. Average improvement was 2.1 grade change over 2 years. Complete suppression of weakness or minimal weakness occurred in 26 of $30(87 \%)$. Complications from prednisone occurred in 17. Azathioprine with prednisone was used in $5(13 \%)$ to achieve better control. Overall incidence of relapse of MG weakness at 2 years was $15.4 \%$.

12 patients underwent thymectomy. Mean age of this group (65.6 yrs) was lower than non-thymectomized patients $(70.1 \mathrm{yrs})$. Thymectomized patients had a better grade improvement ( 2.6 vs 1.6$)$ at 2 years but required a greater level of prednisone than non-thymectomized patients.

This older population of MG patients have a favourable prognosis with immunosuppressive treatment. Complications from therapy are common but can usually be managed satisfactorily.

37.

Evidence for a Temporal Association between onset of Multiple Sclerosis and Canine Distemper in Newfoundland

\section{W. PRYSE-PHILLIPS and G. JONES (St. John's, Newfoundland)}

Outbreaks of canine distemper (CD) have been linked with the subsequent increased incidence of Multiple Sclerosis (MS) on four occasions in northern hemisphere island communities, but the suggested etiologic association has not been reinforced by other avenues of research.

An MS registry has been maintained in Newfoundland for the last three years and with input from the Provincial Government health insurance agency's computerised data base as well as all usual sources, it is considered that ascertainment of MS is reasonably complete, thus allowing eventual determination of regional incidence data. Provincial veterinarians have independently provided information on the dates, regional occurrence and relative severity of outbreaks of $C D$ within the province.
On three occasions since 1968 there has been a temporal association on the Avalon Peninsula between CD outbreaks and increased local MS incidence, as documented by veterinary records and by the MS registry. The lag period between the outbreak of $C D$ and the subsequent increase in local MS incidence was approximately 3 years on each occasion. The association is closer than can be ascribed to chance alone. $(p=<0.05)$. The implications of this finding upon the status of the hypothesis linking MS and CDV will be discussed.

38.

\section{Magnetic Resonance Imaging in Multiple Sclerosis}

W. D. ROBERTSON, D. LI, J. MAYO, S. FACHE, R. A. NUGENT, D. A. GRAEB, J. S. LAPOINTE, D. PATY, S. HASHIMOTO, L. KASTRUKOFF, J. OGER (Vancouver, British Columbia)

Magnetic resonance imaging is excellent in differentiating gray and white matter due to its excellent contrast resolution and its spatial resolution which is approaching that of CT. A series of 60 patients with clinically definite or suspect multiple sclerosis have had magnetic resonance imaging scans. Using a single slice imaging technique, an initial group of 16 patients have had both inversion-recovery $(400 / 1400)$ and spin-echo (40/1080) scans performed in the axial plane. The scans were analyzed as to the total number of lesions present, the size of the lesions, and their distribution in the white matter and periventricular regions. Assessment was also made to see which of the two scanning sequences was more sensitive in determining the presence of abnormality and which would serve as a more sensitive screening procedure.

A second group of 16 patients have had scans performed in the axial and coronal plane using multiple slices in a spin-echo sequence (TE40/1080). Assessment of the number and location of the lesions was again determined and compared to see if the scanning plane influenced the number of lesions detected.

In a second group of 8 patients who have had follow-up MRI scans, assessment of the radiological changes was made, with correlation made with the clinical course.

\section{9.}

\section{Benign Hereditary Chorea: Clinical, Radiological and PET Findings}

O. SUCHOWERSKY, M. HAYDEN, W. R. W. MARTIN, D. K. LI, M. BERGSTROM, R. MARROP, J. ROGERS, C. SAYRE and B. D. PATE (Vancouver, British Columbia)

Benign hereditary chorea $(\mathrm{BHC})$ is a rare neurological disorder characterized by the onset of chorea in infancy or childhood, without associated retardation or dementia. It is inherited as an autosomal dominant trait. Only 12 families with $\mathrm{BHC}$ have been described previously, and no detailed CT, MRI or PET studies are available. We have recently investigated three families. Clinically, the disorder appears heterogeneous and can be divided into two subtypes: Type 1 has delay of motor milestones, with chorea present from infancy. Chorea may improve in adulthood. Type II has normal early development, later onset of chorea in the first or second decade, and slow progression of the movement disorder in adulthood. CT and MRI studies in both types are normal. Preliminary PET findings show decreased activity in the caudate nuclei. BHC is a disorder distinct clinically and radiologically from Huntington's disease. However, PET findings suggest a similar functional abnormality.

\section{0.}

\section{Patterns of Fascicular Involvement in Common Peroneal Neuropathies}

\section{SOURKES and J. D. STEWART (Montreal, Quebec)}

Garland (1952) pointed out that in patients with common peroneal (CP) neuropathies at the level of the knee, there was often considerable 
variation in the motor and sensory deficits. This prospective study was undertaken to more accurately evaluate this phenomenon.

Three muscles innervated by the deep branch of the $\mathrm{CP}$ nerve, tibialis anterior (TA), extensor hallucis longus (EHL) and extensor digitorum brevis (EDB), and two by the superficial branch, peroneus longus (PL) and peroneus brevis (PB) were assessed by concentric needle electromyography for the presence of fibrillations and positive sharp waves. The superficial peroneal sensory action potential (SP-SAP) was recorded with needle electrodes above the fibular neck following distal nerve stimulation.

In 13 patients, the following muscles were denervated: EDB in 12 patients, EHL 11, TA 10, PL 8, PB 9. Denervation tended to be more marked in the muscles innervated by the deep branch. Ten patients had abnormalities of the SP-SAP.

These results suggest that in $\mathrm{CP}$ neuropathies, the fascicles within the deep branch are more frequently (and more severely) affected, although most patients have some degree of involvement of the fascicles to both branches.

REF: Garland, H. and D. Moorhouse. Brit Med J, 2, 1373, 1952.

41.

\section{Follow up Study of Central Infantile Hypotonia}

\section{LESNÝ, and M. PROŚKOVÁ (Prague, Czechoslovakia)}

Central infantile hypotonia being a developmental syndrome appearing in infancy and changing with maturation, 150 infants: 77 boys, 73 girls; were followed up by neurological and psychological examinations. During this follow up 5 diagnostic groups/DG/appeared: DG1: Mental deficiency with developmental disintegration/58/. DG 2: Cerebellar syndrome/29/, DG 3. Minor cerebellar disorder (20), DG 4: Minor brain damage/29/, DG 5: Spastic syndromes. (26) Decrease of hypotonia occurred gradually by successive examinations. The highest degree of hypotonia was in DG 1. Most frequent decrease of hypotonia occurred in DG groups 3-5. There was no significant correlation between the IQ level and the degree of hypotonia.

42.

\section{Sensorimotor Perineuritis - An Autoimmune Disease?}

\section{N. Bourque, B. A. ANDERSON, C. M. DEL CAMPO and} A. A. F. SIMA (Winnipeg, Manitoba)

Following the initial description of sensory perineuritis by Asbury et al. in 1972, no subsequent patients with this disorder have been reported. Asbury's patients demonstrated a painful, distal, sensory neuropathy, and pathological examination of peripheral nerve biopsies revealed focal thickening and inflammatory infiltrates of the perineurium. We report a patient with insidious onset and progressive development of sensorimotor peripheral nerve dysfunction, accompanied by progressive slowing of nerve conduction velocity. Pathological examination of a sural nerve biopsy demonstrated focal thickening of the perineurium, inflammatory infiltrates, and necrosis of perineurial cells. Immunohistology revealed a patchy precipitation of $\operatorname{lgG}$ and IgM on perineurial cells. Ultrastructurally, mononuclear cells were found adjacent to perineurial cells undergoing necrosis. The subsequent clinical course showed gradual improvement following a course of steroid therapy. We suggest that this neuropathy is caused by damage to the perineurial barrier possibly by an immune-mediated destruction of perineurial cells and subsequent compression of the endoneurial content by perineurial scarring.
43.

\section{The Encephalopathy of Sepsis}

\section{A. C. JACKSON, J. J.GILBERT, G. B. YOUNG and C. F. BOLTON} (London, Ontario)

Sepsis occurs frequently in critically ill patients, and may lead to failure of multiple organ systems. The effects of sepsis on the brain have not been systematically studied. Twelve fatal cases with an encephalopathy associated with sepsis were examined in a ten-year retrospective study of 2,172 neuropathologic autopsies. Cases were excluded with other known causes of encephalopathy.

The patients' level of consciousness ranged from drowsiness to deep coma. Six patients had focal neurologic signs; five had seizures. EEGs revealed diffuse or multifocal abnormalities. Although CT head scans and CSF examinations were unremarkable, eight patients had disseminated microabscesses in the brain at autopsy. Three patients had no abnormalities other than mild proliferation of astrocytes and microglia in the cerebral cortex, consistent with a toxic or metabolic effect. Additional findings included cerebral infarcts, brain purpura, multiple white matter hemorrhages, and central pontine myelinolysis.

Sepsis may cause encephalopathy by producing disturbances in cerebral synaptic transmission and cerebral energy production through an endotoxic mechanism. Bacterial invasion of the brain with the formation of disseminated microabscesses, not disclosed by clinical invest igations, is also an important cause of the encephalopathy of sepsis.

44.

Serum Levels of Gamma Aminobutyric Acid (GABA) in Patients with Hepatic Encephalopathy.

\section{G. Y. MINUK, A. WINDER, E. D. BURGESS and E. J. SARJEANT (Calgary, Alberta)}

The cause of the hepatic encephalopathy seen in patients with chronic liver disease is unknown. Recently it has been suggested that gamma aminobutyric acid (GABA), a potent inhibitory neurotransmitter, is pathogenically involved. In this study serum levels of GABA were measured by ion exchange chromatography in: 10 patients with chronic liver disease and hepatic encephalopathy (CLD + HE); 11 patients with chronic liver disease and no evidence of hepatic encephalopathy (CLD); 7 patients with chronic renal failure (CRF); and 10 healthy volunteers. Patients were well matched for age and sex. CLD + HE patients had more advanced liver disease as manifest by the degree of hyperammonemia, hyperbilirubinemia and hypoalbuminemia $(p<0.05)$. Serum GABA levels were elevated in 10/10(100\%) CLD + HE patients, $7 / 11(64 \%)$ CLD patients and $4 / 7(57 \%)$ CRF patients. The mean serum GABA level for CLD + HE patients $(0.92 \pm 0.13 \mu \mathrm{M}$, mean \pm SEM) was significantly greater than the mean for CLD patients $(0.48 \pm 0.05$ $\mu \mathrm{M}, \mathrm{p}<0.05), \mathrm{CRF}$ patients $(0.46 \pm 0.04 \mu \mathrm{M}, \mathrm{p}<0.05)$ and healthy volunteers $(0.39 \pm 0.03 \mu \mathrm{M}, \mathrm{p}<0.001)$. There was no significant difference between mean serum GABA levels in the CLD and CRF patients versus healthy volunteers $(p>0.05)$. These results support the hypothesis that GABA is intimately involved in the pathogenesis of hepatic encephalopathy.

45.

\section{Potential Pitfalls in the Diagnosis of Syringomyelia}

P. C. GATES, J. REM, A. J. FOX and H. J. M. BARNETT (London, Ontario)

Syringomyelia is a rare condition with many underlying predisposing causes. The clinical features are not always classical and thus a high index of suspicion is necessary.

Metrizamide myelography and delayed computerized tomography (CT) scanning are now well established in the diagnosis of syringomyelia. This paper will discuss some of the difficulties that may lead to an erroneous diagnosis. 
The commonest potential cause for a false negative delayed CT scan in patients with syringomyelia is that of timing of the scan after myelography. In any individual, the "optimum" time cannot be predicted. We routinely scan at 6-7 hours and if this is inconclusive, we repeat at 10-11 hours or even 24 hours if necessary. The CT scan may also be negative because of dilution of the contrast within very large cavities or if protracted, vomiting occurs between myelography and CT scanning. A spinal cord tumor may obscure a small associated syrinx.

Linear artefacts, particularly in the thoracic region, may very rarely result in a picture suggestive of syringomyelia and in these circumstances, the "collapsing cord sign" (alteration of the calibre of the cord from the supine to prone position) is helpful.

\section{6.}

Retrogasserian Glycerol Injection In The Treatment of Facial Pain: Preliminary Experience With 20 Patients

\section{FEWER and N. HILL (Winnipeg, Manitoba)}

The definitive treatment of 20 patients with facial pain was attempted using the technique of retrogasserian injection of glycerol. 12/20 patients had trigeminal neuralgia, 6/20 had atypical facial pain and 2/20 had cluster headaches. Median follow-up is 4 months. In 2/20 entry through the foramen ovale was not achieved and no injection was carried out. In $3 / 20$ entry into the subarachnoid space was suboptimal but glycerol was injected and in $2 / 3$ complete relief of pain was achieved while significant partial relief was realized in the other. In 15/20 the contrast study was optimal. Complete pain relief was achieved in 12 , significant incomplete relief in $2 / 20$, and in 1 case no relief was possible. The complications were significant but either of short duration or not severe and will be discussed. They included loss of corneal sensation, cranial nerve palsies and mild "new" pain. Of note is the dramatic relief of all symptoms in the 2 patients with cluster headaches. The technique will be illustrated in detail. We believe it offers significant advantages for both doctor and patient over radio-frequency procedures.

\section{7.}

Deep Brain Stimulation: Technical Problems and How to Avoid Them

\section{K. KUMAR and G. M. WYANT (Regina, Saskatchewan)}

Deep brain stimulation for relief of chronic pain was carried out in 18 patients who underwent 22 implants, with a gratifying pain relief in $80 \%$ of the cases. Notable problems in this series included: electrode displacement during operative procedures 3 , late displacement or fracturing 2, seizures 2 , intracerebral hematoma 1 , short lived effects as disease progressed 2 , undesirable side effects as disease progressed 2 , undesirable side effects namely visual disturbance or paresthesia in a limb during stimulation 2 , wound infection 1 .

The pitfalls can be avoided by adherence to proper patient protocol, including favorable response to Morphine-Naloxone test. The procedure should be carried out under local analgesia to allow physiologic confirmation of the target point and to avoid undesirable side effects. In paraventricular gray implants even $1-2 \mathrm{~mm}$ of change in position can cause a considerable difference in the final outcome of pain relief. Electrodes tend to shift even in the operating room at the time of disengagement and positioning. To prevent late electrode displacement excessive CSF loss at surgery or vigorous scalp massages post operatively should be avoided. Conray ventriculography should be replaced by air ventriculography in cases where multiple attempts at ventriculostomy were made to avoid Conray induced seizure. The electrode should be anchored to the skull with minimal loop in the burr hole to avoid late migration. The burr hole fixation pin should not be used as it may crush and fracture the electrode. This procedure is not advised in malignancy or progressive diseases. An electrode implanted for over 3 months should not be removed as it may precipitate a hematoma.

Changes in hardware design including electrode carrier, anchoring devices, and reinforced electrode sleeve to prevent displacement or fracturing are suggested.
48.

The Effect of Epidural Direct Current Stimulation on Recovery Following Spinal Cord Injury

\section{C. WALLACE, C. H. TATOR and 1. PIPER (Toronto, Ontario)}

The use of direct current (d.c.) stimulation has been shown to enhance spinal cord regeneration after cord transection in the lamprey. The purpose of the present study was to develop the methodology for d.c. stimulation in the rat, and to test its effect on recovery after cord injury.

Twenty-one adult Wistar rats underwent C7-TI laminectomy under pentobarbital anaesthesia, followed by a one minute, 50 gram clip compression injury. Epidural platinum electrodes were placed on the dorsum of the cord and attached to a constant current stimulator placed subcutaneously. The cathode lay distal to the injury site. Control stimulators produced no electrical output, whereas treatment stimulators produced a constant d.c. of 14 uamperes. Animals were tested weekly on the inclined plane for 15 weeks after which the rats were killed, the stimulators tested and the spinal cords examined histologically.

The clinical recovery in those animals receiving d.c. stimulation post-injury was statistically improved over those receiving no stimulation. Inclined plane scores were $28^{\circ} \pm 5^{\circ}$ and $34^{\circ} \pm 4^{\circ}$ for control and treatment groups $(p<.05)$. The correlation between the clinical and histological data will be discussed. Thus, d.c. stimulation of the injured mammalian cord has resulted in a slight, but significant improvement in recovery. In addition, the methodology has been successfully developed for long term d.c. stimulation of the mammalian cord.

\section{9.}

\section{The Effects of Local Cooling on Spinal Cord Blood Flow}

\section{R. R. HANSEBOUT.R. N. LAMONT and V. M. KAMATH(Hamilton, Ontario)}

The internal spinal cord blood flow is measured at the site of local cooling using hydrogen polarography in anesthetized dogs. On cooling, the blood flow decreased to $50 \%$ of the normothermic values during cord cooling to a central temperature of $16^{\circ}$ Celsius. Upon cessation of cooling, internal cord blood flow rapidly returned to within normal values. The implication of the observed phenomena will be discussed relative to the treatment of spinal cord injuries using cooling.

\section{0.}

\section{Fractures of the Odontoid Process: An Analysis of 62 Cases}

M. I. KHAN, R. GRIEBEL and G. STRELIOFF (Saskatoon, Saskatchewan)

Sixty-two patients with a fracture of the odontoid process were treated at the University Hospital in Saskatoon over an 18 year period. The patients' ages ranged from 17 years to 90.7 years, with a mean age of 42.2 years. There were 40 males and 22 females. Cause of the injuries will be examined and related to fracture type and displacement.

Although clinically the exact mechanism of injury was difficult to define, radiological examination showed almost equal members of both flexion and extension injuries. Twelve of the patients had associated spinal cord injuries.

There were 50 type 2 fractures, 10 type 3 and only 2 type 1 fractures. Treatment included one or a combination of the following methodssimple collar or brace, minerva cast, traction with skull tongs, halo traction and vest, anterior and posterior fusions and simple posterior wiring. The complications encountered with these various treatment modalities will be discussed.

Follow up ranged from 1 month to 7.5 years with a mean of 1.4 years. Seven of the 12 patients with cord damage improved, and none of the patients developed new neurological deficits during the course of treatment or follow up.

Successful bony healing and fracture site stability was achieved in $94 \%$ of the patients. Age of the patient and type of fracture are contributing factors to malunion and instability. 
51.

Antero-Lateral Approach for Thoraco-Lumbar Lesions

\section{E. U. EKONG and K. KUMAR (Regina, Saskatchewan)}

The result of antero-lateral approach for thoraco-lumbar spinal lesions in 8 patients is presented. Five patients had burst fractures, while 3 patients had tumors of the thoracic or lumbar spine with anterior encroachment of the spinal canal. The 5 patients with burst fractures had significant neurological deficits, one of whom sustained a complete cord injury at L2. The tumor cases also presented with partial cord lesions. Preoperative investigation revealed more than $50 \%$ compromise of the spinal canal by CT scan or complete myelographic blockage.

The lesions were approached through the bed of the 12 th rib in 7 cases, while thoracotomy was done in one patient with a T9 lesion. Access to the spinal canal was markedly facilitated by cutting and retracting the psoas muscle. After radiologic confirmation of the site, the posterolateral aspect of the vertebral body was removed by the use of a high speed drill. The lesions were then removed from within the spinal canal without cord retraction. An interbody fusion, between the vertebral body above and below, was performed and the psoas muscle repaired.

All the patients showed significant neurological improvement postoperatively. The patient with complete $L 2$ injury recovered sensory and motor functions in the L 3 and $L 4$ roots 6 months postoperatively. There were no complications of this procedure.

The anterolateral approach is a safe and effective way of dealing with lesions, traumatic or neoplastic, in the thoracic or upper lumbar canal where the lesion lies mainly in the anterior spinal canal. The superiority of this technique over laminectomy lies in one's ability to remove the offending lesion without cord retraction.

52.

Full Contact Karate is Just as Dangerous as Boxing: A Biochemical Study

M. SCHWARTZ, A. HUDSON, G. R. FERNIE, K. HAYASHI and R. G. ELGIE (Toronto, Ontario)

It is known that boxers suffer a characteristic, cumulative brain injury from repeated blows to the head. Injury severity correlates well with the length of a boxing career and the number of bouts fought. Much less is known about full-contact karate (kick-boxing) which is relatively new. Full-contact karate resembles traditional karate but the kicks and punches are actually landed, rather than being pulled at the last instant, as in traditional martial arts. Although a combattant can win on points, the surest means of victory is a knock-out. Consequently, fighters strive to land blows to the head. In general, the severity of shearing injury to brain correlates with the violence of acceleration of the head.

To investigate the relative force of kicks and punches, a dummy head was mounted $1.75 \mathrm{~m}$ above the floor (50th percentile man erect) and $1.25 \mathrm{~m}$ (crouch) on a universal joint permitting motion about three axes. The mechanism was contrived to provide constant rotational stiffness about a point corresponding to the C7-T1 level and the head was centered using springs to provide a constant restorative moment about the three axes. The visco-elastic properties of soft tissue were simulated by a mask of plastizote. Fourteen subjects with black belts in karate and two professional full-contact kick-boxers punched and kicked the dummy. Two boxers provided by the athletic commissioner punched but did not kick the dummy. Accelerometer measurements in the 90-120 $\mathrm{g}$ range indicated that safety chops (hand protectors) and safety kicks (foot padding) did not reduce acceleration of the dummy. Tenounce boxing gloves mitigated peak acceleration to some extent. Kicks and punches produced accelerations in the same range.

Violent acceleration of the head by any means produces injury. We conclude that if full-contact karate is widely practised there will soon be cases of kick-boxers' encephalopathy. The authors deplore all activities that encourage head injuries.
53.

The Neurologic Syndromes of Professional Boxers

N. C. HILL (Winnipeg, Manitoba)

Since efforts to ban professional boxing in North America have not been successful, other methods to minimize the neurologic consequences of this activity should be found.

The neurologic syndromes of professional boxers are classified and described as 1) The Concussion Syndromes of Boxers, 2) The Syndromes of Chronic Brain Damage in Boxers, and 3) Acute Brain Syndromes in Boxers. The pathophysiology of these syndromes is discussed.

The physicians' responsibility in recognizing and preventing these syndromes is detailed. The latter includes the education of the boxers, trainers and referees, in recognition of these syndromes by means of a professionally prepared video cassette (which will be demonstrated), by conducting a proper pre-fight examination, and by keeping careful records during (the ring physicians "Score Card") and after the fight. It is hoped that by these means, and others to be suggested, that the harmful effects of professional boxing can be brought to the attention of the boxers themselves; where the responsibility for the prevention and eventual eradication of these injuries finally resides.

54.

\section{Rett's Syndrome}

G. V. WATTERS, B. ROSENBLATT, K. METRAKOS and K. SILVER (Montreal, Quebec)

In 1983 Hagberg et al reported 35 children with a disorder described by Rett in Vienna in 1966. The disorder occurred only in girls, born after a normal pregnancy who at age 7 to 18 months began to deteriorate in their behavior, mental status and language. Autistic features with hand mannerisms in particular occurred over the next 18 months. Ultimately, there was loss of purposeful use of the hands and jerky ataxia of trunk and limbs. Cranial and body growth slowed and functioning stabilized at a low level with later emergence of spasticity and epilepsy. Their EEG records characteristically showed monotonous slow activity in the awake record while in sleep there was much paroxysmal activity with spike wave discharges. Other laboratory studies were normal including CT-Scans, C.S.F. studies, nerve, skin, and muscle biopsies, chromosomal analyses and neurometabolic studies.

We will present 8 such patients with this disorder for the purpose of increasing its recognition and in the hope of increasing its understanding.

\section{5.}

Creutzfeldt-Jacob Disease: Focal Clinical and Unusual Unilateral Epileptiform Discharges

\section{EGGERTSON and N. PILLAY (Winnipeg, Manitoba)}

Initial focal slowing is not uncommon in Creutzfeldt-Jacob disease (CJD), but the occurrence of lateralized or regional periodic epileptiform discharges (PEDS) is unusual.

We have seen three patients presenting with a variety of focal signs indicating lesions of different parts of the brain. Focal or lateralized PEDS were recorded, early in the illness, in all three patients. A 67 year old lady presented with an acute onset of aphasia and coarse pseudoathetotic tremor of the right arm associated with sensory inattention, and other disturbances of cortical sensory function. The EEG showed PEDS in the left parietal-temporal region. The second patient, a 73 year old man, had been dementing for three months. He had a left visual field defect and involuntary tremor of the left arm. PEDS were recorded from the right or independently from the left temporal-occipital area. Progressive dementia and visual hallucinations characterized the presentation in the third patient, a 73 year old male. The focal findings were left visual field neglect, sensory inattention and apraxia. PEDS were recorded mostly from the right occipital or symmetrically from both occipital areas. 
In all three patients, diffuse background slowing with continuous synchronous PEDS over both hemispheres coincided with further progression of the disease. At autopsy none had local brain lesions. The typical histopathological changes of CJD were present in all. Focal or unilateral PEDS, identical to those usually associated with strokes, tumors, or encephalitis may antedate the more classic EEG changes in CJD.

\section{6.}

Severe Familial Encephalopathy in Cree Infants with White Matter Degeneration and Immunological Abnormalities in Cerebrospinal Fluid

D. N. BLACK, E. ANDERMAN, T. COSTA, C. DUMONT, M. E. KABAY, P. KAPLAN, R. NAGY, E. REECE, K. M. VILLEMURE and G. V. WATTERS (Montreal, Quebec)

We report 17 Cree Indian infants in seven closely related sibships. Group I (eight patients) abruptly develop seizures, vomiting, stupor, and spasticity between three and a half and nine months, during a febrile upper respiratory illness or following a typhoid immunization in one patient. This is followed by blindness, fixed pupils, absent tendon reflexes, and death from cardiorespiratory arrest or status epilepticus 10 days to 14 months after onset. CSF showed lymphocytic pleocytosis in one case: protein $58-320 \mathrm{mg} \%$ : CSF IgG is elevated $(3.9-5.6 \mathrm{mg} \%$ ). One patient had a severe combined immunodeficiency. Diffuse sudanophilic degeneration of white matter is present at autopsy.

Group II (nine patients) show progressive microcephaly, quadriparesis, mental retardation and recurrent infections from birth. There is CSF lymphocytic pleocytosis, elevation of protein, and elevation of $\operatorname{lgG}$ (2.2-25.0 $\mathrm{mg} \%)$. Autopsy shows perivascular lymphocytic cuffing. calcifications and demyelinated zones throughout the white matter. One patient showed changes resembling both groups.

Normal biochenical studies have ruled out the known metabolic diseases of the CNS. Adenovirus or CMV has been grown from brain. CSF, or urine in some cases. These two clusters of disease may be attributed to a single autosomal recessive gene, to two independently segregating genes, or to environmental factors interacting with genetic susceptibility to atypical CNS infection in this highly inbred group.

57.

\section{Time Reference System for Prolonged EEG Cassette Recordings}

\section{E. SHWEDYK, R. MORGAN and S. SESHIA (Winnipeg, Manitoba)}

We describe an encoder/decoder system providing an accurate time reference and event marker for prolonged EEG cassette recordings. Digitally encoded amplitude modulation is used for the encoder. Every six minutes a unique eight bit code corresponding to elapsed time is written onto the tape channels. This allows up to 24 hours of time information to be recorded. The eight bit code is represented by $200 \mathrm{~Hz}$ pulse bursts, each burst lasting 1.5 seconds. Event marking is accomplished by a simple momentary switch which encodes a special codeword (hex code FF). The encoding does not interfere with the EEG recording or require an extra channel for recording time information.

The decoder first separates the EEG signal from the time reference/ event marker information. A majority of the channel outputs must be valid to produce a time update or an event indication. This redundancy provides reliability against time information loss due to artifact noise. The outputs are presented to a micro-processor system programmed to interpolate between the 6 minutes time codes. This permits a continuous time display and can simultaneously display an event occurrence. To preserve accuracy the interpolation algorithm is continually adjusted by the recorded time information.

The described time reference/event marker adds the capability of correlating clinical events with prolonged EEG recordings enhancing the usefulness of prolonged recordings.
The authors would like to acknowledge the support of the Children's Hospital of Winnipeg Research Foundation, Health and Welfare, Canada and NSERC.

58.

\section{Morphology of Spikes in Spike-Wave Complexes}

\section{W. T. BLUME and J. F. LEMIEUX (London, Ontario)}

Bilaterally synchronous spike-wave complexes recorded referentially from two 16 channel grids centered frontally on the scalp were analysed by an automated morphological analysis program. The morphology (slope, amplitude, duration) of the spike 2 component was studied as was the symmetry of these parameters between the first and second half-waves (FHW and SHW). The spike-wave complexes of nine patients was studied.

We have already demonst rated that bilateral spike-waves complexes usually originate unilaterally and propagate contralaterally (Lemieux and Blume, EEG Journal, 1983).

The morphology of the originating spikes differed from those appearing at sites of propagation. Propagated spikes were more asymmetrical than were originating spikes. The amplitude and duration of second half-waves of propagated spikes usually exceeded those for first halfwaves for both originating and propagating spikes but the inter halfwave differences were greater for propagated spikes.

These morphological differences support the thesis of Musgrave and Gloor (Epilepsia, 1980) that the physiology of initiating spikes differs from that of propagating spikes in apparently bilaterally synchronous spike-wave complexes.

\section{9.}

Use of Subdural Electroencephalography in Candidates for Surgical Relief of Uncontrolled Partial Epileptic Seizures

W. T. BLUME, J. P. GIRVIN, R. S. MCLACHLAN and D. C. JONES (London, Ontario)

Although depth electroencephalography has become widely used in an attempt to delineate the origin of partial (focal) epileptic seizures, its limitations and risks are being recognized (Gloor, Epilepsy International Symposium, 1983).

In those patients in whom clinical analysis and scalp and sphenoidal EEG recordings have failed to determine the origin(s), we have used subdural electroencephalography as an alternative to depth recordings.

The five patients whom we selected from subdural recordings had uncontrolled partial seizures whose origin appeared from interictal and ictal EEGs as well as clinical analysis to be predominantly unifocal. However, sources of doubt about origin from scalp electroencephalography included: 1) multiple independent spike foci, 2) ambiguous origin of seizures, and 3) an apparently unusual pattern of seizure spread.

Four to eight strings of very flexible platinum rings on a silicone substrate were inserted into appropriate areas of both hemispheres through burr holes. Each string contained seven electrode positions, each at $2 \mathrm{~cm}$. intervals. Recordings were carried out for 7 to 14 days.

Recorded seizures originated in the previously suspected hemisphere in each instance. However, the origin and pattern of spread could be more clearly delineated and this influenced the site of subsequent surgical resection in such instances. In one case, subdural recordings revealed a mesial frontal ictal onset which was in-apparent on scalp recordings.

Thusfar, we have not encountered any complications in these five patients who have undergone six implantations (one patient twice). 
The ultimate value of subdural recordings will depend upon: 1) their safety in a greater number of patients, and 2) whether the additional information obtained was instrumental in effecting a surgically related clinical improvement. Our numbers are too small and follow-up too short to determine this now.

60.

\section{EEG Activation by Alfathesin During Electrocorticography}

\author{
F. E. LeBLANC, M. A. LEE and J. R. MALTBY (Calgary, Alberta)
}

Alfathesin is a non-barbiturate anaesthetic induction agent which was released in Canada in 1977. It is a mixture of two steroids, Alphaxaline and Alphadolone. Our attention was drawn to its possible use during epilepsy surgery by one sentence in an article in the British Medical Journal (17:9:77, p. 736) referring to work by Comelli (Proc. VI World Congress of Anaesthesiology 1976). Comelli noted the enhancement of epileptiform EEG discharges during Alfathesin induction of anaesthesia for cerebral angiography in patients with cerebral tumors and previously documented epileptiform EEG abnormalities. He suggested its possible use in subanaesthetic doses for EEG activation. Since 1979 we have used Alfathesin for EEG activation during electrocorticography in 17 patients. No adverse effects have occurred. The usual induction dose of Alfathesin is $75 \mathrm{uL} / \mathrm{kg}$ or $5 \mathrm{ml}$ in the average adult. Based on Comelli's suggestions and our previous experience with Methohexital (Brietal) we have used $10-20 \%$ of the induction dose $(0.5-1.0 \mathrm{ml})$ of Alfathesin. At this dosage there is little or no alteration of the patient's level of consciousness but enhancement of the epileptiform abnormalities occurs within 20-45 seconds. In 16/17 patients epileptiform activity was either initiated or markedly enhanced. In two cases we compared the effects of Brietal and Alfathesin in the same patient. In one case, surgery was carried out under general anaesthesia and Alfathesin served to activate a previously silent epileptogenic focus. In our experience Alfathesin is a safe and reliable activator of epileptiform activity during electrocorticography.

\section{1.}

\section{Topographical Analysis of the Centrotemporal Discharges in} Benign Rolandic Epilepsy of Childhood

\section{L. GREGORY and P. K. WONG (Vancouver, British Columbia)}

The morphology and topographic distribution of the centrotemporal discharges in 10 patients with Benign Rolandic Epilepsy of Childhood (B.R.E.C.) were studied.

All patients exhibited clinical and EEG features characteristic of typical B.R.E.C. as described by Lombroso (1967) and Lerman (1973). Eight patients had a unilateral centrotemporal focus and 2 patients had independent bilateral foci (total of 12 foci studied). Twenty individual discharges were averaged for each focus and this "averaged spike" was then transformed into a series of topographical maps. The voltage from the 19 routine $10-20$ electrodes was displayed on a head diagram and formed the basis from which all remaining points were computer generated using linear interpolation. The completed voltage contour maps were displayed chronologically, separated by $2 \mathrm{msec}$ in time.

The result was the identification of a stereotypic dipole field present along the rolandic region in all discharges. During the peak of this dipole the negative pole was maximum at the centrotemporal region with the positive pole involving the bifrontal region. The onset of the discharge was not identical in all foci, with 3 different onset types identified. The duration of the dipole ranged from $16-46 \mathrm{msec}$ (mean 28 $\mathrm{msec}$ ). The offset was simultaneous in 5 discharges and in the remaining 7 discharges the frontal region led to temporal region in returning to the baseline.

It is hypothesized that all discharges arise from a single generator which is orientated tangential to the surface. Most likely this generator is situated in the lower rolandic region where the zero potential exists, between the frontal positivity and centrotemporal negativity.
Identification of this dipole configuration may be useful for differentiating B.R.E.C. from focal epilepsy of other etiologies.

62.

\section{EEG May Predict Response of Absence Seizures}

H. Z. DARWISH, V. LANGE, H. B. SARNAT and R. H. A. Haslam (Calgary, Alberta)

The response of 40 children with absence seizures was reviewed. Only children of normal intelligence and an EEG showing serial general. ized spike and wave (SW) or polyspike and wave (PSW) dysrhythmia were included. There were 15 boys and 25 girls and the age at review was $11 \pm 4$ years. The age at onset of absence seizures was $7.5 \pm 2.5$ years. All children had been followed a minimum of 6 months and $70 \%$ were followed for over one year. 19 children had simple absence (SA) and 21 had complex absence (CA). 14 children (7 with SA and 7 with CA) had a history of one or more generalized tonic clonic seizures (GTC), four occurring with fever, and usually antedating onset of absence seizures. The EEG showed SW in 12 and PSW in 28. Random focal or multifocal spikes or sharp waves (FS) were present in 27 who had PSW and 6 who had SW. (FS) with SW occurred usually with complex absence seizures. In patients with PSW EEG, FS occurred equally in simple or complex absence. Phenobarbital (PB) was the initial treatment in 11 children, valproic acid (VA) in 6, and ethosuximide (ESM) in 7.8 had initial trials with phenytoin (PHT) and 4 with carbamazepine $(\mathrm{CBZ})$, reflecting the presence of $\mathrm{FS}$, and past history of GTC seizures. A total of 93 courses of treatment were pursued for the 40 children. $39 \%$ were completely controlled (no reportable absences and no paroxysms of generalized SW or PSW). $36 \%$ were significantly improved (no reportable absences; EEG still shows generalized SW or PSW). 24\% continue to have clinical absence. At present, 21 are on VA and are controlled, 8 are on ESM with 5 controlled. 21 had a trial of ESM with 16 failing to respond, with 7 then tried on VA with complete control. The VA level needed to control SA was $483 \pm 70 \mathrm{umol} / \mathrm{L}$, whereas that to control CA was $605 \pm .47 \mathrm{umol} / \mathrm{L}(\mathrm{p}<0.01)$. Absence seizures associated with the presence of focal epileptiform discharges in the EEG were more likely to respond to valproic acid than to ethosuximide $(\mathrm{p}<0.05)$.

63.

\section{Serum Free and Total Valproate Concentrations in Children}

K. FARRELL, F. S. ABBOTT, J. M. ORR, P. K. WONG and J. .E. JAN (Vancouver, British Columbia)

Valproic acid (VPA) is a highly protein-bound drug whose free fraction may be affected by free fatty acid concentration, comedication and certain diseases. Thus its clinical effect might be expected to correlate better with the free rather than total VPA concentration in the serum. Methods of measuring serum free VPA concentration are now commercially available but there have been no clinical studies which have examined the indications for its use. We studied healthy children on VPA monotherapy (25) and VPA polytherapy (17). We measured serum total and free VPA concentrations, using equilibrium dialysis and gas liquid chromatography, and SGOT in blood drawn in the fasting state and before the morning dose.

In children on monotherapy, a correlation was found between VPA dose/kg/day and both serum free VPA $(P<0.001)$ and total VPA $(P=0.016)$ concentrations. In contrast, children on polytherapy showed a poor correlation. The serum total VPA concentrations correlated well with the serum free VPA concentrations in both groups $(P<0.001)$. SGOT levels correlated with VPA dose $/ \mathrm{kg}$ in both groups but not with total or free concentrations. These data show that the dose/kg/day is a poor predictor of serum free or total VPA concentration in children on polytherapy. The good correlation between total and free VPA concentrations suggests that monitoring of free VPA concentrations should be limited to those situations where VPA binding is likely to be affected. 
64.

\section{Clobazam in Children with Intractable Seizures}

K. FARRELl, J. E. JAN, J. V. JULIAN, T. A. BETTS and P. K. WONG (Vancouver, British Columbia)

Clobazam is a benzodiazepine with anti-epileptic activity and relatively mild sedative side-effects. Its use in the management of intractable seizures has been described largely in adults. We describe the use of clobazam as adjunctive therapy in 16 children whose seizures were refractory to all other anti-epileptic drugs. The children, aged 2 to 16 years, had infantile spasms, Lennox-Gastaut syndrome or partial seizures. Clobazam was added to the existing drug regimen and any benzodiazepine comedication was withdrawn over one month. The initial dose was 2-5 $\mathrm{mg} /$ day and this was increased at 5 day intervals up to a maximum of 32 $\mathrm{mg} /$ day. Response to therapy was evaluated monthly using seizure count done by a parent, EEG, a side-effect questionnaire, blood count and tests of coagulation, liver and renal function.

Four (4) patients are seizure free and 5 have had a reduction in seizure frequency greater than $50 \%$. Other beneficial effects were a marked improvement in behaviour and reduction in drowsiness (11), decreased drooling (4), improved appetite (3) and improved sleep pattern (3). Adverse side-effects included increased drowsiness and disturbed sleep pattern in one patient, and ataxia and mild depression in another. Blood count, coagulation studies, liver enzymes and renal function remained stable during therapy. Thus clobazam, used as adjunctive therapy or to replace another benzodiazepine, may be effective in reducing seizure frequency and lessening side-effects in children with intractable seizures.

\section{Poster Presentations}

65.

\section{CT Imaging of the Cranial Nerves}

T. K. MASARY, P. J. SWEENEY and M. T. MODIC (Cleveland, Ohio)

Cranial nerve palsies are a common symptom of neurological disease. The charge of neuro-imaging is to determine in the least invasive fashion if a structural tesion is responsible. This exhibit will illustrate the various imaging modalities which can directly visualize the cranial nerves or the pertinent surrounding anatomical structures. This will include high resolution CT with and without intravenous and/or intrathecal contrast, digital subtraction and conventional angiography and nuclear magnetic resonance. The normal anatomy and optimal modality for specific regions will be demonstrated. Relative examples of cranial nerve dysfunction, the clinical presentation and the responsible structural pathological alteration will be depicted.

66.

\section{Myasthenia Gravis, Thymoma and the Anterior Junction Line}

\section{P. J. SWEENEY and P. O'DONOVAN (Cleveland, Ohio)}

In the management of myasthenia gravis there is agreement that the presence of thymoma is an indication for thymectomy. Estimates of the prevalence of thymoma in myasthenia range from a low of $8 \%$ to as high as $20 \%$. Diagnostic efforts to determine the presence of tumor are primarily radiographic and range from PA chest films with oblique views to mediastinal tomography and $C T$ of the mediastinum. These procedures are expensive and, summated, can exceed $\$ 600$ in cost. In approximately $30 \%$ of the general population, involution of the thymus is associated with the development of a radiographic "marker" - the Anterior Junction Line (AJL). To visualize this structure establishes contiguous upper lung borders and lack of any tissue between them; the
AJL is effaced by any soft tissue such as adenopathy or thymoma. Armed with knowledge of this useful radiographic detail, the neurologist is in an excellent position to "visually dissect" the mediastinum on his myasthenic's PA chest films, in search of peccant tumor. To visualize these coapting lung borders (the AJL) establishes, with considerable assurance, the absence of thymoma and offers the patient both financial savings and roentgen exposure.

This presentation will illustrate and elaborate on the $\mathrm{AJL}$, its development and how to visualize and determine its presence.

\section{7.}

The Rate of Blood Pressure Elevation is a Crucial Factor in the Induction of Recurrent Intracranial Hemorrhage in an Experimental Model

\section{E. ARBIT, A. GILLICH and S. BREM (Montreal, Quebec)}

A potential factor in the pathogenesis of recurrent intracranial hemorrhage $(\mathrm{ICH})$ from rupture of cerebral berry aneurysm for example, is the acute elevation of arterial blood pressure (ABP). In a rat model where an $\mathrm{ICH}$ was created, we found that the rate of rise in blood pressure could predict the onset of recurrence. Male Sprague-Dowley rats were anesthetized, paralyzed, artificially ventilated, and maintained normoxemic normocapneic and normothermic. The middle cerebral artery was surgically divided, resulting in an $\mathrm{ICH}$ which was allowed to cease spontaneously with thrombus occlusion. In a control group, which was observed without ABP manipulation, no recurrent $\mathrm{ICH}$ occurred for as long as an hour. In a second group, infused with metaraminol, ABP was raised rapidly at a mean rate of 30 torr $/ \mathrm{min}$ Within 2 minutes all animals had a recurrent hemorrhage occurring at a mean pressure increment of 56 torr. In the third group, ABP was elevated gradually at a mean rate of $5 \mathrm{torr} / \mathrm{min}$. Again, a recurrent hemorrhage occurred in all animals, but in this group it occurred within 15 minutes of ABP manipulation and a mean pressure increment of 85 torr was tolerated $(p<0.02)$. Recurrence of ICH developed at differing ABP levels for individual rats. There was no absolute safe level; however, a slow incremental rate permitted tolerance of much higher levels of $A B P$ elevation. If we can extrapolate these results to the clinical situation, mild hypertension or gentle elevation of ABP in order to maintain an adequate perfusion pressure may be tolerated in patients with $\mathrm{ICH}$ with minimal risk of hemorrhage recurrence.

\section{8.}

\section{Prognostic Profiles in Acute Stroke}

B. R. CHAMBERS, J. W. NORRIS, B. L. SHURVELL and V. C. HACHINSKI (Toronto, Ontario) (London, Ontario)

Improving the immediate and long-term prognosis after stroke requires the identification of factors influencing outcome. Nine hundred and sixty-seven patients with acute cerebral infraction, admitted to the MacLachlan Stroke Unit, were followed prospectively for up to seven years to identify prognostic determinants during the acute and chronic phases.

Cumulative survival was $80 \%$ at one month, $75 \%$ at one year and $50 \%$ at five years. Increased mortality during the first 30 days correlated with stroke-related factors: depressed level of consciousness $(p=0.0000)$, leg weakness $(p=0.0000)$, arm weakness $(p=0.0000)$ and lateral gaze deviation ( $p=0.000)$, and antecedent factors: increasing age $(p=0.0000)$, cardiac disease $(p=0.0005)$ and previous stroke $(p=.0040)$, as observed on admission. Survival was best predicted by the patient's age and level of consciousness. Long-term mortality correlated with disability on hospital discharge $(p=0.0000)$, age $(p=0.000)$, heart disease $(p=0.0000)$ and was greater in carotid as opposed to vertebrobasilar stroke $(p=.0004)$. The sex of the patient, hypertension, diabetes and the side of the lesion had no effect on short- or long-term survival.

We conclude that short- and long-term survival after cerebral infarction depends on a patient's age and prior health, and on factors related to the stroke itself. Survival may be accurately predicted at the time of admission. 
69.

\section{Improvement in the Retinal Circulation Following Extracranial- Intracranial Bypass Surgery}

\author{
M. STANDEFER, J. R. LITTLE and R. TOMSAK (Cleveland, \\ Ohio)
}

The retinal circulation of 29 patients, age 30 to 71 , (median 60 years) with symptomatic internal carotid occlusion (23) or severe inaccessible stenosis (6) was studied both before and after superficial temporal to middle cerebral artery bypass surgery (STA-MCA) using intravenous fundus fluorescein angiography (IVFA) and ophthalmodynamometry (ODM). Retinal artery pressures (RAP) were abnormal preoperative in 20 of 21 patients and improvement in RAP's for the ipsilateral eye was noted postoperatively in 16 patients. RAP's obtained during the late postoperative period ( 3 months) were significantly improved when compared to preoperative values. IVFA values were abnormal in 18 of 19 patients preoperatively and improvement was noted in 15 patients postoperatively. In contrast to RAP's, IVFA values were significantly improved during the early postoperative period ( 3 months) for both eyes. Preoperative patients with normal fundus exams (10) had significantly better values for IVFA and ODM than patients with venous statis retinopathy (VSR). The findings of the study suggest that STAMCA anastomosis may be beneficial in the treatment of retinal ischemia secondary to severe inaccessible carotid artery stenosis or occlusion. STA-MCA bypass appears to improve the retinal circulation by augmenting collateral input. This may result in the reversal of a vascular steal between the retinal and the cerebral circulations with an improvement in occular perfusion.

70.

\section{Multiple T.I.A.'s, Lupus Anticoagulant and Verrucous Endocarditis}

\section{J. G. D'ALTON, D. N. PRESTON, J. BORMANIS, M. S. GREEN} and G. KRAAG (Ottawa, Ontario)

Lupus anticoagulant (L.A.), an anticardiolipin antibody, is present in some patients with systemic lupus erythematosus (S.L.E.) and occasionally with other conditions. While prolonging the PTT, it is associated in vivo with thrombotic events, including cerebrovascular disease. An unusal case is described of a young woman with probable S.L.E. and a circulating L.A., who had onset of multiple T.I.A.'s several years after a major cerebral infarct.

At age 19, while on the pill, she had a left hemispheric infarct, middle cerebral branch occlusion on angiography and a prolonged PTT with negative ANA. She subsequently had no new neurological symptoms until age 23, but then for over 3 years had recurrent stereotyped T.1.A.'s consisting of left amaurosis fugax several times daily, with less frequent vertigo and alternating hand numbness. She had a positive ANA, low serum complement, a lupus anticoagulant and echocardographic evidence of verrucous endocarditis. Her symptoms persisted with Prednisone $30 \mathrm{mg}$. daily, but stopped when Aspirin was added. The pathogenesis will be discussed.

\section{1.}

\section{New Stereotaxic Instrument for Use with Cat Scan, Digital Angiography, NMR and Pet Scan}

\section{A. OLIVIER, G. BERTRAND, T. PETERS and V. TIPAL (Montreal, Quebec)}

The dramatic changes that have taken place in the field of neuroimaging have also brought about a completely new approach in the field of stereotaxic surgery.

In order to use the new facilities to their maximum, the characteristics of the stereotaxic frame to be used become of the utmost importance.
Since most of these scanning techniques are not available in the operating suite, the frame to be used must be compact and of light weight so that, once fixed to the skull, the patient can be brought to the various facilities outside the operating theatre.

The other characteristic of the frame essential for maximum use of these procedures is the ease and facility with which it can be replaced in the very same position to the skull at different sittings.

We have devised a stereotaxic instrumentation which, we think, fulfills these criteria. Interchangeable fiducial marker plates can be readily installed for the various stereotaxic imaging procedures so that subsequent cross correlations can be carried out. Furthermore, the apparatus can use both the Talairach orthogonal and the Leksell spherical systems. The procedures of depth electrode placement and biopsy of brain tumours are used to illustrate the principle of computer stereotaxic imaging and the various components of the apparatus $\left({ }^{* *}\right)$

** apparatus built by TIPAL INSTRUMENTS LTD (Montreal, Canada).

\section{2.}

\section{Ischemic Cerebral Mitochondrial Lipid Changes}

\section{R. F. DEL MAESTRO and C. H. VIERECK (London, Ontario)}

Mitochondrial dysfunction has been related to the development of ischemic cell death. In order to investigate the effect of ischemia at the membrane level, mitochondria lipid analyses from normal and ischemic rat brains were undertaken. The rat model of complete cerebral ischemia used involved sacrificing the animals by decapitation and placing the brains in a nitrogen bubbled $37^{\circ} \mathrm{C}$ saline bath. Ischemic periods of 15 and 30 minutes were studied.

Preliminary findings show that total phospholipid phosphorous (per mg protein) does not change during ischemia. Resolution of phospholipids by one dimensional thin-layer chromatography and determination of individual phospholipid phosphorous (per $\mathrm{mg}$ protein) however, shows a $19 \%$ decrease in cardiolipin at 15 minutes and a $15 \%$ drop after 30 minutes of ischemia. Phosphatidyl ethanolamine, phosphatidyl choline, phosphatidyl serine, phosphatidyl inositol and sphingomyelin levels showed no significant changes. Total cholesterol levels increased $21 \%$ at 15 minutes and $26 \%$ at 30 minutes of ischemia.

The above may be indicative of cholesterol induced fluidity changes in the outer mitochondrial membrane and degradation of cardiolipin in peripheral phospholipids of mitochondrial enzymes and may increase our understanding of events occurring in the cerebral mitochondria during ischemia. Further lipid analyses of free fatty acids, phospholipid fatty acids as well as mitochondrial membrane fluidity changes are in progress.

Supported by the Ontario Heart Foundation Grant 3-26

73.

Intracerebral Haemorrhage following Ingestion of Oral Catecholaminergics: Arteriographic Changes

\section{A. J.STOESSL, J.TURNBULL and G. B. YOUNG (London, Ontario)}

We report two cases of serious intracerebral haemorrhage occurring in young women following their first use of oral medications containing catecholaminergic preparations (phenylpropanolamine and ephedrine or pseudoephedrine). Both women were previously well, and there was no evidence for systemic vasculitis, coagulopathy, aneurysm or arteriovenous malformation. Angiography in both cases, performed approximately forty hours following drug ingestion, revealed the beading pattern typical of that seen in previously reported cases of presumed amphetamineinduced 'vasculitis'.

We feel that the arteriographic abnormalities are non-specific, and should not be construed as necessarily indicative of arteritis. Alternate possibilities are presented. 
74.

The SHARP Imaging System - In Vivo Imaging of Cerebral Metabolism in the Experimental Animal

\section{C. HOWSE, B. T. McKEE and M. HOGAN (Kingston, Ontario)}

Positron Emission Tomography is a major technological advance in neurology providing the ability to measure metabolic and physiologic functioning of the brain in-vivo. We have developed a new type of imaging system known as SHARP using planar detectors based on the multi-wire proportional chamber. This sytem can achieve resolution of $4 \mathrm{~mm}$ FWHM. Imaging studies to be reported have been carried out on the lightly anaesthetized rhesus monkey or cat, using [18F] 2-fluoro-2deoxy-D-glucose (kindly provided by Drs. L. Yamamoto and M. Diksic).

Images were reconstructed in 16 slices. The cerebral cortex, basal ganglia, brain stem, cerebellum and spinal cord were clearly demonstrated in the monkey brain, which is approximately half the size of the newborn human brain. These preliminary studies demonstrate the feasibility and value of this approach, particularly for achieving high resolution in relatively small volumes.

75.

Neurological Dysfunction and Brain Neurotransmitter Levels in the Gerbil Stroke Model: Effect of Naloxone, Morphine and Clonidine

D. P. J. BOISVERT, M. WALLEY, G. BAKER, T. NIHEI, and T. DEWHURST (Edmonton, Alberta)

The opiate antagonist naloxone is currently under investigation as a therapeutic agent in acute stroke. We studied the acute effects of unilateral carotid occlusion on neurological status and brain levels of noradrenaline (NA), dopamine (DA) and gamma-aminobutyric acid (GABA) in gerbils. During a 3 hour period following carotid occlusion, animals with neurological deficits, seizures or no symptoms were treated with naloxone, clonidine or morphine respectively. In each case, a control placebo (saline) treated group was measured.

Neurological deficits such as paralysis were unaffected by naloxone: Morphine produced neurological deterioration in asymptomatic animals while, in contrast, clonidine aborted post-ischemic seizure activity. In control animals decreases in dopamine and increases in GABA and NA were observed in ischemic hemispheres. These changes were most pronounced in animals with seizures and could be reversed by clonidine. Neither morphine or naloxone produced significant neurotransmitter alterations.

The results of this study do not support a role for naloxone in the treatment of stroke. Clonidine abortion of ischemic seizures with inhibition of neurotransmitter changes may be of clinical significance and warrants further study.

76.

\section{Effect of Sympathetic Stimulation on Carotid Stump Pressure}

\section{P. J. BOISVERT (Edmonton, Alberta)}

Wide variation in carotid stump pressure (CSP) measured during carotid endarterectomy or carotid occlusion for intracranial aneurysm is usually attributed to differences in vascular resistance across the Circle of Willis. This study was performed to determine whether sympathetically induced increases in external carotid artery resistance also affect CSP. Measurements were performed during normotension and normocapnia in anesthetized cats $(n=5)$ and monkeys $(n=6)$ with common carotid artery occlusion.
Sympathetic stimulation produced an increase in CSP of $30 \pm 2 \mathrm{~mm}$ $\mathrm{Hg}$ (mean $\pm \mathrm{SEM}$ ) in monkeys and $27 \pm 6 \mathrm{~mm} \mathrm{Hg}$ in cats. The increases in CSP were accompanied by marked increases in carotid pulse pressure. In monkeys, external carotid artery occlusion resulted in an increase of CSP of $33 \pm 7 \mathrm{~mm} \mathrm{Hg}$ and abolished the CSP response to sympathetic stimulation, thus confirming that the increase in CSP during stimulation was due to a selective increase in external carotid artery resistance.

The results of this study demonstrate that the level of sympathetic activity plays an important role in determining CSP and may account for much of the variation in CSP observed clinically. Sympathetically induced increases in CSP and pulse pressure may contribute to sustained or increased size of intracranial aneurysms following therapeutic carotid ligation.

77.

Near-Occlusion of Extracranial Blood Vessels in Osteopetrosis

G. J. V. MAKIN, R. K. COATES, D. PELZ, C. DRAKE and H. J. M. BARNETT (London, Ontario)

Osteopetrosis is a very rare disease of osteoclasts resulting in hard brittle bones and bone marrow failure. Although often fatal, prolonged survival in the autosomal dominant variety occurs. Common neurological features include palsies of the first eight cranial nerves, usually ascribed to bony overgrowth of skull foramina. In some cases, however, plain $\mathrm{x}$-rays of the optic foramina in blind patients failed to demonstrate stenosis. Another occasionally reported, but poorly understood, observation has been dilated scalp veins. We report several previously undescribed radiological features of osteopetrosis. Severe stenosis of the internal carotid arteries occurred in two patients. with well-developed vertebrobasilar and ophthalmic collaterals. Injection of a dilated scalp vein in an affected child revealed non-filling of the posterior part of the superior sagittal sinus, the transverse sinuses, and the internal jugular veins. The dilated scalp veins appeared to shunt blood directly from the anterior and middle parts of the superior sagittal sinus to the jugular vein. Computerized tomography (CT) in a blind osteopetrotic adult, whose plain films of the optic foramina were normal, demonstrated patent but abnormally long optic canals. In this case, the optic nerve may have been stretched rather than compressed. The carotid canals could not be demonstrated by CT in a patient with internal carotid stenosis.

78.

Headache and Visual Loss - "Pseudo-Giant Cell Arteritis"

K. M. HOYTE, B. D. MCLEOD, C. J. PENNEY and W. J. BECKER (Calgary, Alberta)

Giant Cell Arteritis (GCA) is a rare disorder affecting older patients. Typically it presents with unilateral headache and may be associated with visual loss due to ischemic optic atrophy.

We have recently investigated two patients who presented with headache and visual loss and a third who presented with localized temporal headache. They were all initially felt to have GCA. Temporal artery biopsies, when performed, were negative. The patient's headaches all responded dramatically to corticosteroid administration. In one patient, the vision improved as well.

Subsequent neurologic investigation demonstrated neoplasms in all three patients. One was a pituitary adenoma, another a subfrontal oligodendroglioma, and the third a frontal glioblastoma.

All patients had surgical treatment with some improvement.

We would like to use these cases to illustrate the difficulty of diagnosing GCA in some patients and to reaffirm the classic clinical features of GCA. We would also like to stress that steroid responsive headache with visual loss is not necessarily due to GCA. 
79.

\section{Spontaneous Dissection of the Carotid Artery in Association with Fibromuscular Dysplasia}

\author{
D. ATACK, M. EDEN-WALKER, B. BENOIT and G. BELANGER \\ (Ottawa, Ontario)
}

We present a unique case of spontaneous dissection of the internal carotid artery in association with radiological evidence of fibromuscular dysplasia in the same and opposite cerebral arteries. (photographs)

Radiological evidence of spontaneous resolution is shown (photograph) and the implications this poses for management are discussed.

80.

\section{The Murine Ependymoblastoma: Response to Radiation Treatment}

\section{P. J. MULLER and K. H. SHIN (Toronto, Ontario) (Calgary, Alberta)}

We have assessed the effect of radiation therapy on normal and subcutaneous tumor bearing $\mathrm{C} 57 \mathrm{~B} 1 / 6 \mathrm{~J}$ mice. The radiation was administered by an Eldorodo Co-60 telatherapy unit at a dose rate of 307 $\mathrm{rads} /$ minute and a source to surface distance of $50 \mathrm{~cm}$. The mice were irradiated 5 at a time; 10 mice were used for each radiation dose.

Whole body irradiation of normal mice with a single fraction, 5 daily fractions (conventional fractionation, CF) or 3 fractions per day for 5 days (multiple daily fractionation, MDF) resulted in LD50 values of 947,1365 and 1534 , respectively $(\mathrm{p}<0.01)$.

Tumor growth retardation was assessed in mice after the implantation of $5 \times 10^{6}$ tumor cells into the thigh muscles. The time in days for the tumor to cause a $50 \%$ increase in the size of the thigh of the treated mice minus the time for a $50 \%$ increase in the control mice was defined as the growth retardation time (GRT). The first day of treatment was designated day 1 . For the CF groups at total doses of 1000, 1250, 1500, $1750,2000,2250$ or 2750 rads the GRT were $5,8,15,15.5,25.5,32.5$, and 89, respectively. For the MDF groups at the same total doses the GRT were $6,6.5,15,32.5,16.5,93.5$, and 31 , respectively. There was a clear linear relationship between GRT and total dose for the CF groups $(y=0.0428 X-49.279, r=0.894, p<0.025)$ but not for the MDF groups $(y=0.0290-23.085, r=0.574$, N.S. $)$.

We conclude that MDF causes less lethal toxicity than CF in this model but were unable to demonstrate a consistent prolongation of GRT in the MDF groups compared to CF.

81.

\section{Carbon-Dioxide Laser in Neurosurgery}

\section{K. KUMAR and C.E.U. EKONG (Regina, Saskaskatchewan)}

The $\mathrm{CO}_{2}$ laser is an important adjuvant to the neurosurgical armamentarium. Our experience with the 35 watt laser machine over the past six months is presented. During this period 27 craniospinal procedures were performed with the help of this laser. These consisted of meningiomas 10 (supratentorial 5 , infaratentorial 4 , spinal 1 ), acoustic neuromas 2 , astrocytomas 4 (cerebrum 3 , cerebellum 1), metastatic intracranial tumors 4 , neurofibroma of the spinal cord 1 , intramedullary tumor of the spinal cord 1 , myelotomy or commissurotomy 2 , drez lesions 2 .

The $\mathrm{CO}_{2}$ laser in our experience has proved to be a superior tool because of safety, precision, control of blood loss and minimal retraction needed at surgery.

It was found to be particularly useful in the removal of the meningiomas of the posterior fossa and base of the skull, acoustic neuromas, myelotomies and in excision of intramedullary tumors of the spinal cord. It was of no specific benefit in astrocytomas, metastatic, and soft suckable tumors of the brain. The laser was found to be inferior to conventional technique in highly calcified tumors or very vascular tumors such as hemangioblastomas or vascular meningiomas. Drez lesions could not be effectively made with this equipment due to the fact that the depth of the repetitive lesion varied due to tissue density and moisture content.

So far we have not tried using the laser for pituitary tumors or disc surgery.

Videostrips of selected cases will be presented.

82.

Effects of Hyperthermia on BCNU Cytotoxicity Against BCNUResistant 9L Rat Brain Tumor Cells

\author{
V.F. DA SILVA, P.H. GUTIN and D.F. DEEN (San Francisco, \\ California)
}

Even though it is the most effective agent against malignant brain tumors, 1,3-bis (2-chloroethyl)-1-nitrosourea (BCNU) used alone or in combination with other agents cannot produce long-term remission or cure, presumably because of problems inherent in drug delivery or because of the presence or de novo production of BCNU-resistant cells. While various approaches to modified therapies with BCNU have been tried, none have been successful.

It has been shown that, because of the Arrhenius relationship for reaction rates, the number of reactive species produced by hydrolysis of $\mathrm{BCNU}$ is increased at higher temperatures; therefore, at higher temperatures BCNU is more cytotoxic to cells. This suggests that hyperthermia could be used to advantage in combination with BCNU. We treated both BCNU-sensitive and BCNU-resistant $9 \mathrm{~L}$ rat brain tumor cells with BCNU at five temperatures between 42 and $44^{\circ} \mathrm{C}$. The effect of hyperthermia alone on both cell lines was the same. Treating both cell lines with $\mathrm{BCNU}$ at temperatures above $37^{\circ} \mathrm{C}$ caused a progressive increase in cell kill; thermal enhancement ratios for the sensitive cells ranged between 1.4 and 1.7 , and for the resistant cells between 3.2 and 8.8. The increase in cell kill for the sensitive cells was no greater than would be predicted from values calculated using a drug-dose concept for the increased number of active BCNU species produced at higher temperatures, which implies that factors other than increased dose were responsible for the increased cell kill. However, the increase in cell kill for the resistant cells was more than the value calculated for an increase in active species only. At temperatures above $43^{\circ}$, the cell kill of resistant cells was equivalent to the cell kill that BCNU caused in sensitive cells at $37^{\circ}$. These results suggests that $\mathrm{BCNU}$ and hyperthermia combination protocols might be used to advantage in a clinical setting.

83.

\section{TSH-Secreting Pituitary Adenoma}

F.B. MAROUN, N. FARID and J.C. JACOB (St. John's, Newfoundland)

A 32 year old woman was treated for hyperthyroidism with radioactive iodine. In retrospect, she had an "inappropriately" normal TRH stimulation test before treatment; when she subsequently became hypothyroid, TSH diminished from $7.7 \mathrm{mu} / \mathrm{L}$ to $3.4 \mathrm{mu} / \mathrm{L}$. She was treated with Thyroxin $0.1 \mathrm{mg} /$ day. TSH initially increased to $282 \mathrm{mu} / \mathrm{L}$, fell to $36.8 \mathrm{mu} / \mathrm{L}$, and again increased above $240 \mathrm{mu} / \mathrm{L}$ when the dose of Thyroxin was increased to $0.2 \mathrm{mg}$. Her serum TSH and prolactin increased markedly after stimulation with TRH. During T-3 suppression test, TSH initially increased to $78 \mathrm{mu} / \mathrm{L}$ on day 3 and diminished at day 7 to $60 \%$ of basal. In addition to these studies, radiologic diagnostic procedures were performed, and a diagnosis of TSH secreting pituitary adenoma was made. Tissue submitted for EM and immuno-cysto chemistry following transphenoidal surgery showed that she had TSH secreting cells and large pituitary cells with prominent rough endoplastic reticulum which did not stain with any pituitary hormone - specific antibody. The adenoma recurred after 9 months, though she remained asymptomatic. This case provided us with an opportunity to study responses of TSH secreting tumor to positive and negative physiological stimuli. 
84.

\section{Needle Biopsy of Intracavernous Lesion in an Unusual Case of Painful Ophthalmoplegia}

\section{D.W. ROWED, E.E. KASSEL and A.J. LEWIS (Toronto, Ontario)}

Fine needle biopsy, guided by computerized tomography (CT) is useful in selected intracranial lesions. This technique may be safely and effectively employed in relatively inaccessible sites such as the cavernous sinus.

An otherwise healthy 82 year old man presented with progressive, painful right ophthalmoplegia. Initial CT demonst rated a small contrastenhanced area at the orbital apex, contiguous with the superior orbital fissure. Other investigation which included cerebral angiography was not helpful. Despite high dose cortico-steroids the patient's pain worsened, ophthalmoplegia progressed and vision began to deteriorate in the right eye. Open biopsy via lateral orbitotomy of the progressively larger area of contrast enhancement at the orbital apex was nondiagnostic.

Serial CT's defined a $5 \mathrm{~mm}$. mass in the anterior cavernous sinus. This was biopsied transcutaneously via the medial superior orbital fissure by a technique which will be described, yielding a definitive and unexpected diagnosis of aspergillosis.

Preliminary experience suggests that this approach might be safely and productively employed in unusual cases of inflammatory or neoplastic disease within the cavernous sinus.

\section{5.}

Chemical versus Bacterial Meningitis after Intrathecal Methotrexate - Can CSF Cultures be Trusted?

\section{G.J.V. MAKIN (London, Ontario)}

Meningitis may follow administration of intrathecal methotrexate. Though most often due to chemical irritation, this diagnosis often cannot be made with certainty. Many patients are, therefore, treated empirically with antibiotics, covering the wide variety of opportunistic pathogens possible in an immunosuppressed patient shortly following instrumentation of the subarachnoid space. The decision to discontinue antibiotics is often based on CSF culture results. Methotrexate, a drug closely related to trimethoprim, may interfere with these cultures and could lead to a false-negative report. I have tested this hypothesis with several micro-organisms by monitoring in vitro growth in the presence of methotrexate and/or folinic acid (leucovorin). Both growth rate and final growth of $S$. aureus and $S$. fecalis are inhibited by methotrexate in clinically relevant concentrations. Inhibition of $S$. fecalis is partially reversed by folinic acid. E. coli is inhibited to a lesser degree. $P$. aeruginosa, an organism not normally sensitive to diaminopyrimidines, is unaffected by methotrexate. These studies underline the need to consider factors other than the results of CSF culture alone when making the decision to discontinue antibiotics in this setting. I feel that folinic acid $(1 \mathrm{mg} / \mathrm{ml})$ should be added to the culture medium in this situation.

86.

\section{Microvessel Assessment in a New Experimental Glial Model}

\section{FARRELL and R.F. DEL MAESTRO (London, Ontario)}

The interaction between neoplastic cells and endothelial cells is poorly understood. In neoplasms of glial origin, the presence of malignant glial cells appears to induce abnormalities in the growth and differentiation of host tissue vessels that leads to microvessel proliferation and subsequent vasogenic edema. The objective of these studies was to characterize angioarchitectural and functional differences between the microvessels of experimental glial tumours and peritumoural normal tissue.

The $\mathrm{C} 6$ astrocytoma cell line has biochemical and pathological similarities to the most common malignant glial tumour, glioblastoma multiforme. The injection of $\mathrm{C} 6$ astrocytoma cells directly into the cerebral hemisphere of neonatal Wistar rats was inadequate since a well-demarcated interface was not always present.
Spheroids of $\mathrm{C} 6$ astrocytoma cells were grown to $400-500 \mathrm{um}$ in size. Each spheroid contains $30-40,000$ cells and when placed into the cerebral hemisphere of adult male Sprague-Dawley rats results in a reproducible model in which a spheroidal geometrical growth continues and a distinct tumour - normal tissue interface can be studied. Alkaline phosphatase staining in conjunction with microangiography has allowed quantitative angioarchitectural studies of such parameters as microvessel size, length and density. Functionally Evans blue extravasation begins to occur 9 days post-implantation which suggests that the permeability characteristics of these vessels alter with continued growth. The combining of the two technologies and use of adult Sprague-Dawley rats appear to have resulted in a good model to assess tumour microvessel growth.

\section{7.}

Scalp Necrosis Complicating Preoperative Embolization of Vascular Meningiomas

\section{R.C. CHAN and G.B. THOMPSON (Vancouver, British Columbia)}

The authors report two patients who suffered ischemic necrosis of their scalps following preoperative therapeutic embolic occlusion of the external carotid artery and its terminal branches for highly vascular meningeal tumors. Local anaesthesia, selective catheterization of the external carotid artery, and radio-opaque polyvinyl alcohol and gelfoam as embolic agents were used. The various complications of external carotid artery embolization have been reviewed. To avoid inadvertent reflux of nonopaque emboli into the internal carotid artery, fluoroscopic control to ensure rapid antegrade flow of contrast material and patency of the external carotid artery is required. The use of a low pressure injection technique with frequent test injections will minimize the risk of neurological complications. The importance of preserving normal branches of the external carotid artery mainly superficial temporal artery during embolization, and careful design of the scalp flap to avoid ischemic necrosis are recommended.

88.

Leptomeningeal Metastases from Inflammatory Carcinoma of Breast with pure CSF Polymorphonuclear Leukocytosis

D.R. MACDONALD, M.J. INWOOD and P.E. COOPER (London, Ontario)

Metastases of systemic cancer to the leptomeninges typically produces a mononuclear lymphocytic pleocytosis in the cerebrospinal fluid (CSF). We recently saw a patient with carcinomatous meningitis in whom a pure CSF polymorphonuclear leukocytosis caused initial diagnostic confusion. A 32 year old woman with "inflammatory" poorly differentiated infiltrating ductal carcinoma of the breast, metastatic to regional lymph nodes, treated with mastectomy and chemotherapy, presented with 10 days of increasingly severe generalized headache and 2 days of intermittent nausea and vomiting. She was afebrile. The initial examination revealed no neurologic abnormalities. Bone scan and $C T$ brain scan were normal. Over 3 days she became obtunded and developed a right facial weakness. A lumbar puncture revealed clear CSF with opening pressure $40 \mathrm{~cm}$ water. CSF analysis showed WBC $334 \times 10^{6} /$ litre (100\% PMN's), protein $560 \mathrm{mg} /$ litre and glucose 1.2 $\mathrm{mmol} /$ litre. She was thought to have bacterial meningitis and was treated with intravenous antibiotics without response. CSF gram stain, india ink and cultures were negative. Repeat lumbar puncture, 2 days later, again produced clear CSF under markedly increased pressure. CSF analysis showed WBC $118 \times 10^{6} / \mathrm{L}$ (68\% PMN's, 32\% lymphocytes), protein $720 \mathrm{mg} /$ litre and glucose $1.8 \mathrm{mmol} /$ litre. Cultures were again negative. CSF cytology on both samples was subsequently reported as positive, containing many malignant cells consistent with metastatic breast carcinoma. She improved transiently with dexamethasone but died before definitive therapy could be given. Permission for autopsy was refused. Leptomeningeal metastases may occasionally induce a marked CSF polymorphonuclear leukocytosis causing diagnostic con- 
fusion between meningeal carcinomatosis and infective meningitis. In cancer patients a high index of suspicion should be kept for leptomeningeal metastases as diagnosis may be difficult and untreated disease progress rapidly.

89.

\section{Cisplatinum Chemotherapy for Recurrent Brain Tumors}

\section{D.R. MACDONALD, J.G. CAIRNCROSS, M.R. RATHBONE and L. JENKS (London, Ontario)}

Seventeen patients with recurrent malignant brain tumors (glioblastoma 11 , anaplastic astrocytoma 5, medulloblastoma 1) were treated with cisplatinum (cis-diaminedichloroplatinum -II) chemotherapy. All had received prior radiation therapy and $\mathrm{BCNU}$ chemotherapy. Cis platinum, $60-100 \mathrm{mg} / \mathrm{m}^{2}$ each $4-6$ weeks for $1-10$ courses, was given intravenously with hydration and high-dose metaclopramide as anti-emetic. Acceptable toxicity included mild reduction of creatinine clearance requiring dose reduction in I patient, high-frequency hearing loss in I and mild nausea or vomiting in all. Myelosuppression was minimal and required no dose adjustment. One patient developed massive cerebral edema, herniation and died. The hydration regime was subsequently changed with no further problems. Of 10 patients evaluable to date, $2(20 \%)$ had unequivocal partial responses by clinical and/or CT scan criteria, 3 (30\%) had stabilization of disease for 2-6 months and 5 had disease progression. One responder is currently alive, working full-time, I5 months from onset of cisplatinum therapy. The other responder has suffered hearing loss following the first course of treatment. Cisplatinum has some activity against malignant brain tumors but has potential serious toxicity. Further studies with newer, less toxic platinum analogs are needed.

\section{0.}

\section{Focal Facial Spasms with Cerebellar Tumor}

\section{P.B. JAVAKAR and S.S. SESHIA (Winnipeg, Manitoba)}

Involuntary facial movements can result from lesions of the cerebral cortex, basal ganglia, facial nucleus or facial nerve. We describe a one year old child with focal tonic-clonic facial spasms, who had a cerebellar tumor, a lesion hitherto not associated with such phenomena. Each paroxysm began with ptosis and twitching of the left eyelid. The right eyelid and left angle of the mouth were involved within seconds. Frequently, there would then be upward deviation of the eyes, decreased responsiveness, respiratory and chewing movements. The episodes waxed and waned, movements occurring 1 to $3 / \mathrm{sec}$. at their peak. They lasted three seconds to 30 minutes, were almost constant whilst awake but rare during sleep. There was no response to anticonvulsants or to Haloperidol. Neurological examination was normal except for a mild inco-ordination and impaired parachute response in the left upper limb.

Several EEGs were unremarkable but rhythmic theta activity accompanied a few episodes; spindles were less marked on the left. BAEP were normal. C.T. scan showed a left cerebellar mass. This was respected and found to be an astrocytoma.

The features, in our case, are in keeping with experimental data and with clinical observations in adults, that attribute a responsible role for the cerebellum in paroxysmal motor activity and EEG changes. A cerebellar cause should be considered in children with involuntary motor phenomena.

91.

\section{Hemifacial Spasm (HFS) in Childhood}

\section{G.M. RONEN, J.R. DONAT and A. HILL(Saskatoon, Saskatchewan)}

Hemifacial spasm is characterized by involuntary, irregular twitching of muscles supplied by the facial nerve. It occurs almost exclusively in adults; only two cases have been reported in children.
Our patient is a nine-year-old female who presented with an 18-month history of intermittent right HFS. The spasms begin with involuntary eyelid closure followed by repetitive eyelid twitching and facial spasm with pulling of the mouth. The spasms can be triggered by facial movements. The spasms have been documented on videotape. There is no history of prior facial nerve palsy or injury. Physical examination is otherwise normal. Pure-tone audiogram, brainstem auditory evoked potentials, EEG, CT scan, skull $x$-ray, tomograms with special views of the internal auditory and facial nerve canals were normal. Stimulation of each supraorbital nerve caused blink reflexes with distal latency of $10.0 \mathrm{~ms}$ and amplitude of $0.6 \mathrm{mv}$ on each side. Stimulation of the right supraorbital nerve resulted in synkinesis of the ipsilateral facial muscles. The HFS remained unchanged the following six months. The parents refused treatment with carbamazepine.

In cases of HFS in which a cause is established, pulsatile compression by arteries in the posterior fossa is the most common. Tumors, aneurysms and arteriovenous malformations are rare causes. In our patient the normal findings suggest a nonprogressive etiology. This case report indicates that, contrary to generally held opinion, HFS may occur in the pediatric age group.

92.

\section{Cortical Visual Impairment in Children}

S. WHITING, J. JAN, P.K. WONG, O. FLODMARK, K. FARRELL and A.Q. McCORMICK (Vancouver, British Columbia)

Fifty children with severe permanent cortical visual impairment referred to the British Columbia Children's Hospital Visual Impairment Program are presented.

The clinical features are emphasized and certain diagnostic criteria are redefined. Several clinically distinct subgroups emerged, for example, shunt failure, perinatal hypoxia with occipital infarction, and cerebral dysgenesis.

The relative usefulness of the electroencephalogram, CAT Scan, traditional visual evoked response, and a new technique, visual evoked potential mapping, is analysed.

This multidisciplinary study indicates that the current definition and concepts of cortical blindness have many limitations when applied to children and should be modified.

93.

\section{Relaxation Training for Pediatric Migraine}

P. HUMPHREYS, P. McGRATH, J.T. GOODMAN, P. FIRESTONE and D. KEENE (Ottawa, Ontario)

Migraine is a frequent disorder in children, affecting approximately $4 \%$ of the population. When migraine is severe and frequent it may interfere with school and social activities. Very few controlled studies evaluating treatments for pediatric migraine have been published.

This paper reports on a randomized control trial evaluating the effectiveness of individualized relaxation training compared to a waiting list control and a non-specific, attention-control treatment. The subjects were 120 children between 9 and 17 who were diagnosed as having migraine and recorded an average of at least one headache per week. Following a four week baseline, children were stratified on the basis of severity of headache and randomly assigned to treatment. Each child had six weekly sessions with a trained therapist. The major dependent variables were derived from a daily headache diary.

Results indicate that all groups had reduced headache following treatment and at three month follow up. However, relaxation training was statistically and clinically superior to both of the control groups for children with more severe headache. 
94.

\section{Serial VEP Recording in Hydrocephalus}

\section{S.G. COUPLAND and D.D. COCHRANE (Calgary, Alberta)}

While steady-state ERG and VEP abnormalities have been reported in adult patients with raised intracranial pressure (ICP), it has typically been observed in the presence of papilloedema. Papilloedema is an uncommon feature of hydrocephalus in infants with open cranial sutures making the reliable diagnosis of raised ICP more difficult. In those patients with possible shunt malfunction delay in surgical intervention can result in serious life-threatening sequelae. Consequently, the prompt recognition of elevated ICP is of critical importance. We have attempted to assess such patients at risk for shunt malfunction by recording transient and steady-state flash VEPs both prior to and on a regular basis following ventriculoperitoneal shunt insertion. In addition, in several patients sequential VEP recordings were obtained over a 24.48 hour period where continual ICP monitoring was obtained. Normalization of VEP peak latencies following lowering of ICP through shunt insertion was observed in the majority of the patients. In those patients requiring repeated shunt revisions the VEPs were observed to increase in peak latency with documented increases in ICP. Power spectral distribution of the steady-state VEP was also found to change systematically with variations in ICP. Repeat VEP recording was found to provide assistance in the investigation and management of hydrocephalic infants with suspected shunt malfunction.

95.

Hereditary Motor Sensory Neuropathy Type I (HMSNI) Presenting as Scapuloperoneal Atrophy (Davidenkow's Syndrome): Electrophysiological and Pathological Studies

G.M. RONEN, N. LOWRY, A. HILL and H.B. SARNAT (Saskatoon, Saskatchewan) (Calgary, Alberta)

Scapuloperoneal weakness may be myopathic or neurogenic. Most neurogenic cases are considered due to anterior horn cell disease. Rarely, scapuloperoneal weakness may represent a variant of HMSNI (Davidenkow's syndrome). This syndrome is not distinct genetically and has occurred in a patient whose sibling had classical HMSNI, i.e. Charcot-Marie-Tooth disease.

We report a 14-year-old male with weakness and wasting in a scapuloperoneal distribution, areflexia, distal hypoesthesia, ataxia, intention tremor, kyphosis and pes cavus. Nerve conduction was diminished, and an EMG demonstrated neurogenic features. Histological studies of muscle demonstrated marked fibre-type grouping and atrophic fibres. Electron microscopic studies of sural nerve demonstrated a decrease of large myelinated fibres and onion-bulb formation. The parents and four siblings are unaffected clinically. Nerve conduction studies were borderline in the father.

Our case could be sporadic, or inherited as an autosomal recessive or autosomal dominant condition in which case the father would represent a forme fruste. The clinical features in our patient suggest Davidenkow's syndrome. Detailed electrophysiological and pathological studies indicate HMSNI. These studies confirm the contention of Davidenkow and others that weakness and wasting in a scapuloperoneal distribution combined with distal hypoesthesia may be due to HMSNI.

96.

Lumbar Canal Stenosis Causing Neurapraxia Along Root Fibers; Its Recognition by EMG Examination

A.J. WILBOURN, M.R. HANSON and J.R. LITTLE (Cleveland,
Ohio)
Lower extremity weakness caused by lumbosacral root compression Lower extremity weakness caused by lumbosacral root compression
is sometimes due to conduction block (neuropraxia), rather than axon- degeneration, occurring along the motor root fibers. Recognition of this condition is important, since prognosis is excellent (rapid, complete recovery), if surgical decompression is done before (possible) progression to axon-loss occurs. While this situation cannot be detected by clinical examination, it can be by EMG studies, as the following report illustrates.

A male, aged 70, experienced progressive leg weakness, with right foot-drop, over a 5-week period, without sensory or sphincter difficulties. Clinical impression was peripheral polyneuropathy, but EMG examination revealed fibrillations in multiple L5-innervated muscles bilaterally, consistent with a cauda equina lesion. Myelography showed severe lumbar canal stenosis. Although the right tibialis anterior (TA) muscle was clinically weak (3/5-MRC), and contained almost no voluntary motor unit potentials (MUPs) on needle examination (NE), the motor conduction amplitude recorded over $i t$, while stimulating peroneal nerve, was normal (7.0 Mv vs $6.5 \mathrm{Mv}$ on left). The latter excluded significant axon-loss having occurred and suggested that the foot-drop was due to neuropraxia at the root level. 6 days after extensive lumbar laminectomy the right TA was significantly stronger (4.5/5), and normal numbers of MUPs were present in it on NE, confirming the EMG impression.

\section{7.}

\section{Evoked Potentials in Fisher's Syndrome}

\section{N. PILLAY and S. OLIVER (Winnipeg, Manitoba)}

In 1956, Fisher described what he believed to be a variant of the Guillain-Barre syndrome. It consists of ophthalmoplegia, ataxia and areflexia. Opinions differ on whether the triad of signs is due to the disease of the central or peripheral nervous systems, or both. While pathological changes are found in the peripheral nerves, similar evidence in the neuraxis is lacking.

Electrophysiological studies were performed on two patients with Fisher's syndrome. Peripheral nerve conduction studies were abnor$\mathrm{mal}$ in both. The PI00 latencies on pattern reversal visual evoked potentials (VEPs) were significantly and asymmetrically prolonged with preservation of the amplitudes. Brainstem auditory evoked responses (BAERs) reflected central dysfunction with normal absolute wave I and prolonged interpeak latency (I-V) in one patient. The VEPs and BAERs returned to normal, but somatosensory evoked potentials (SEPs) remained delayed with dispersed responses, and reduced amplitudes in both patients when seen at five and ten months after the onset of the disease.

The abnormal nerve conduction velocities suggested a partial conduction block in the peripheral nerves. The same mechanism could account for the SEP delays from the spinal cord and cortex. It is impossible to explain the abnormalities of the brainstem and visual pathways without implying a different pathogenesis. These findings support both central and peripheral locations for the lesions in Fisher's syndrome.

98.

The Use of Intraventricular Metrizamide to Demonstrate an Ependymal Cyst

\section{R. BLOM and N. WITT (Edmonton, Alberta)}

Intraventricular cysts are rare and rarely become symptomatic. We report the case of a thirty year old male with chronic headache, investigated by CT scan and intraventricular metrizamide, in whom an ependymal cyst was found in the left lateral ventricle and which did not communicate with the ventricle. As intrathecal metrizamide rarely adequately enters the lateral ventricle, this technique may prove to be the optimal method of preoperatively demonstrating the extent of similar unenhancing intraventricular lesions. 
99.

\section{Intracranial Abscess: A Thirty Year Experience}

\section{D.A. HERNANDEZ and R.F. DEL MAESTRO (London, Ontario)}

One hundred and eight consecutive cases of intracranial abscess diagnosed between 1952 and 1982 have been reviewed. The number of cases related to direct extension remained constant while cases resulting as a complication of trauma, craniotomy and systemic infections increased. The high mortality associated with post-traumatic, postoperative and metastic brain abscesses and the increasing evidence of these over the last ten years has kept the overall mortality high $(25 \%)$. Follow-up was obtained with a mean of 44 months in 80 survivors. Thirty-three per cent of the survivors had residual motor deficits, $24 \%$ had alterations in their mental status and $28 \%$ developed seizures; $73.3 \%$ were able to return to work, $71.4 \%$ were able to return to school and $13 \%$ were left with behavioural problems.

Early diagnosis, intensive antibiotics/surgical treatment and stabilization of the general medical conditions continue to be critical in the management of intracranial abscess.

100.

\section{Interhemispheric Subdural Hematoma}

N.A. RUSSELL, B.G. BENOIT, R. DEL CARPIO, G. BELANGER and K.B. MALLYA (Ottawa, Ontario)

Current literature perpetuates the erroneous belief that interhemispheric subdural hematomas are very rare. This reflects the difficulty in diagnosis which existed prior to the advent of computorized tomographic scanning. Our experience with 9 cases that were encountered over a 12 month period at the Ottawa Civic Hospital stimulated a review of the literature.

Eighty-three cases were obtained, only 10 of which had been reported prior to 1974. The hematoma usually results from trauma, but has been reported following aneurysm rupture and in association with anticoagulant therapy and blood dyscrasias. It results in certain characteristic neurologic abnormalities that include contralateral crural paresis, hemiparesis with the leg being weaker than the arm, seizures involving the contralateral leg, and paraparesis.

CT scanning provides precise diagnosis, the hallmark of which is a crescent shaped posterior midline density, the flat medial border of which is formed by the falx and the convex lateral border by the hematoma bulging into the ipsilateral hemisphere.

Approximately 50 per cent of these hematomas are small and resorb spontaneously. The larger masses must be removed and parasagittal craniotomy, never burr holes, is the only safe and acceptable method.

The features of our cases correspond to those described in the cases reported.

101.

\section{Head Injury Rehabilitation}

M.D. COLLINS (Toronto, Ontario)

Head injury is a major source of adult disability. The incidence of head injury is $250 / 100,000$ population, and of these patients, approximately two thirds will require rehabilitation.

This paper will discuss the head injury rehabilitation program offered at the acute hospital (Sunnybrook Hospital) and the rehabilitation hospital (The Riverdale Hospital). From January, 1983 to December, 1983, 44 people were treated at The Riverdale Hospital ( 8 are still in hospital, and 3 left before the initial asessment was completed). On admission, $48 \%$ were severely disabled, $46 \%$ moderately disabled and $6 \%$ minimally disabled. The average length of stay for those patients was 69 days. Of the 33 patients who stayed in the program, $93 \%$ were able to return home.
This would seem to indicate that rehabilitation does favourably affect the outcome, however, as many variables would have to be taken into account, further attempts must be made to validate the program. Precise physical levels will be given as well as changes in cognitive scores using the W.A.I.S., I.Q., Memory Quotient, and WoodcockJohnson test data.

102.

\section{An Unusual Cause of Torticollis}

K. BURTON, R. KIM, P. McGUINESS, J. McFARLAND, and D.B. CALNE (Vancouver, B.C.) (Vancouver, Washington) (Vancouver, British Columbia)

We report a 4 year old child who presented with acute torticollis, without prior drug exposure, trauma, or febrile illness. There were no accompanying neurological abnormalities. A sedative "cocktail" briefly eliminated torticollis, but atypically, no improvement during sleep was observed, and transient torticollis in the opposite direction also appeared. Conventional radiology showed no evidence of cranio-cervical dislocation, however CT scanning of the cervical spine under general anaesthesia demonstrated rotatory atlanto-axial subluxation.

This cause for torticollis is not described in the general literature of movement disorders. Most patients are diagnosed by orthopaedic surgeons, and conservative management, employed shortly after onset, is usually successful.

We present a videotape of the patient manifesting torticollis, discuss criteria for the clinical and radiological diagnosis of fixed rotatory atlanto-axial subluxation and comment on it s treat ment and prognosis.

103.

The Effect of Naloxone on Spinal Cord Blood Flow and Cardiac Output Following Spinal Cord Injury

\section{M.C. WALLACE and C.H. TATOR (Toronto, Ontario)}

The narcotic antagonist, naloxone, has been shown to improve spinal cord blood flow (SCBF) and clinical recovery following experimental spinal cord injury. The purpose of this study was to determine the effect of naloxone on SCBF, cardiac output and blood flow to other organs following clip compression injury. Wistar rats were anaesthetized, paralysed and ventilated, with mean systemic arterial pressure (mSAP) and arterial blood gases recorded continuously. Animals underwent C7-T1 laminectomy and a 1 minute 65 gram clip compression injury at T1. Radioactive microspheres were used to calculate SCBF, cardiac output and blood flow to other organs 15 minutes following injury, and after a 1 hour treatment period. Treatment animals received a bolus of naloxone $(10 \mathrm{mg} / \mathrm{kg}$ ) followed by a one hour intravenous infusion ( 2 $\mathrm{mg} / \mathrm{kg} / \mathrm{hr}$ ).

After injury, there was no significant difference in mSAP between control and treatment groups. Naloxone failed to improve posttraumatic SCBF in the segment of injured cord or in other regions of the cord. Naloxone did not prevent the progressive decline in cardiac output seen between 15 and 75 minutes after injury. Thus, this study does not support previous claims for the beneficial effect of naloxone on posttraumatic SCBF.

104.

Hand Wasting in Cervical Spondylitic Myelopathy

A.E. GOODRIDGE, T.E. FEASBY, C.F. BOLTON, G.C. EBERS and W.F. BROWN (London, Ontario)

Three patients, ages 41-68, presented with hand weakness and were found to have hand wasting secondary to cervical spondylitic myelopathy. The duration of symptoms ranged from 2 years to 8 years. No patient had radicular pain but all patients had a significant degree of wasting of all intrinsic hand muscles (unilateral in two and bilateral but asymmetri- 
cal in one) and various degrees of forearm weakness and wasting. Only one patient had long tract signs. Myelography revealed extradural cord compression secondary to spondylosis at the C3-4, C4-5 and C5-6 levels in 1 patient, at C4-5 and C5-6 in one and at C5-6 in one. The lower cervical intervertebral foramina were normal. EMG showed motor unit loss in the clinically affected muscles in all 3 cases.

The phenomenon of hand wasting associated with mid-cervical lesions is probably similar to that seen in foramen magnum lesions and may be due to cord ischemia distal to the compression. This was thought to be due to impaired venous drainage rather than anterior spinal artery compression. This clinical syndrome often leads to diagnostic confusion. Spondylitic myelopathy, therefore, is often dismissed from consideration because of the absence of long tract signs and the relative infrequency of root involvement at $\mathrm{C} 8$ and $\mathrm{Tl}$.

This study emphasizes the occurrence of hand wasting in patients with spondylitic lesions in the mid-cervical region.

105.

\section{Serratus Anterior in C7 Radiculopathy}

\section{G.J.V. MAKIN, G.C. EBERS and W.F. BROWN (London, Ontario)}

Lesions of the seventh cervical root are common and cause a readily recognized neurological syndrome. Recognition of this syndrome is essential in differentiating $\mathbf{C} 7$ root lesions from lesions of the brachial plexus or peripheral nerves. Serratus anterior weakness is not generally included in this syndrome. We report five cases of $\mathrm{C} 7$ radiculopathy (surgically verified in four), in which weakness of the serratus anterior was present in addition to the usual findings. This was manifest as winging of the scapula, exclusively during the unusual action of pushing forward with the hands at waist level. Photographic demonstration of this sign, contrasted with scapular winging in lesions of the long thoracic or spinal accessory nerves, will be provided. Explanation of this sign involves recognizing that the serratus anterior is a highly segmented muscle supplied by at least three spinal segments (C5, C6 and $\mathrm{C7}$ ). Vector analysis of this clinical finding complements anatomic evidence suggesting that the powerful lower digitations of the muscle may be primarily supplied by the $\mathrm{C} 7$ root in some cases. Clinically, the finding of scapular winging is not inconsistent with a C7 radiculopathy, as it was initially concluded to be in two of our cases.

\section{6.}

\section{Paraplegia without Radiographic Abnormality in an Adult}

\section{M.L. LONG and K.M. HUNTER (Calgary, Alberta)}

Paraplegia without fracture is a well recognized post traumatic complication in children. It is, however, rare in adults. We present a case history and literature review of this entity.

\section{7.}

\section{Extreme Lateral Disc Protrusions}

\section{J.D. McQUEEN and G. STRELIOFF (Saskatoon, Saskatchewan)}

Lateral disc protrusions have been well known for 50 years; similarly lateral stenosis has been recognized for 30 years. We wish to present cases of extreme lateral soft disc protrusions where the pathology was invisible on myelography and in some instances at surgery as well. Examples illustrated better accuracy with computed tomography as compared with myelography in lumbar disc disease.

A collection of 58 patients with evidence of lumbar disc disease were studied. They were seen during 1982-83, at University Hospital, Saskatoon or Saskatoon City Hospital. Of these 58, 3 patients had extreme lateral disc herniation which was picked up on computed tomography but not myelography. All three of the herniations were at the L5-SI level.
In two instances the clinical findings indicated lateral nerve root involvement, when correlated with the CT results. It is suggested that, in the past, the group may have constituted one small portion of the patients with failed back syndromes.

108.

Pitfalls In Epidural Spinal Cord Stimulation for Chronic Pain Relief

K. KUMAR, G.M. WYANT, C.E.U. EKONG, (Regina, Saskatchewan)

Epidural stimulation by chronically implanted electrodes was carried out under local analgesia in 32 patients. In 17 of these cases, pain was secondary to failed back syndrome. Patients were followed from six months to four years with a gratifying pain relief in $74 \%$ of the cases. The notable complications in this series included wound infections 3 ; electrode displacement 4 ; electrode fracturing 2; failure to negotiate optimum segment 1 ; CSF leak 1 ; and fibrosis at the stimulating tip in one patient.

In the literature $25 \%$ of the patients regress in their pain relief in the first year due to various pitfalls in technique and hardware. These pitfalls can be avoided by proper patient selection protocol, including initial favorable response to TNS stimulation and subsequent verification of pain relief by temporary percutaneously implanted electrodes. In order to select the proper level of stimulation site and number of electrodes to be implanted it is imperative that the implant be done under local analgesia with intraoperative stimulation. Electrode displacement should be recognized early by a change in the pattern of stimulation and invariably needs readjustment. Cessation or severe dampening of stimulus suggests electrode fracturing or fibrosis at the stimulating tip and may require electrode replacement. Wound infection usually has cleared on suitable antibiotics and rarely needs removal of the hardware.

A change in electrode design by way of a retractable spike for better fixation, an adjustable anchoring sleeve to prevent fracturing, and multiple contact stimulating tip are suggested to optimize results.

109.

The Clinical Relevance of the Sleep Deprived Electroencephalogram

\section{G.M. KLEIN and M.A. LEE (Calgary, Alberta)}

There are many techniques available to provoke epileptogenic activity in the electroencephalogram (E.E.G's) of patients who are believed to suffer from seizure disorders, but who have normal waking EEG's. Amongst these techniques, that of the sleep deprived EEG is in wide usage. Although this method is undoubtedly safe, it is time consuming. The purpose of this retrospective study was to evaluate the value of the sleep deprived EEG in three groups of patients, all of whom had had normal or nonspecifically abnormal waking EEG's. One group had definite witnessed seizures, and the purpose of the repeat EEG's was to elicit epileptogenic activity. One group had probable but unwitnessed seizures and the clinician involved could not make a definite diagnosis of epilepsy. The final group had symptoms unlikely to represent seizures. The percentage of sleep deprived groups was compared. We were able to confirm the value of a sleep deprived EEG in selected patients.

110.

Co-Occurrence of Temporal Spikes and Generalized Spike Wave Discharges

R.M. SADLER and W.T. BLUME (St. John's, Newfoundland)(London, Ontario)

Among patients who were candidates for temporal lobe resection because of intractable complex partial seizures we encountered some who had, in addition to their focal temporal epileptiform potentials, 
sporadic generalized spike wave discharges. To investigate this phenomenon further a computerized EEG classification system was used to retrospectively identify 19 patients (from a population of 10,400 ) who had demonstrated both temporal spikes and generalized spike wave discharges, although not necessarily during the same EEG recording. Previous candidates for temporal lobe resection who had had many EEGs (including sleep readings) were chosen as controls. We thus minimized the chance of missing spike wave discharges on the basis of recording time. Clinical information (age, sex, seizure type(s), age of onset of habitual seizure disorder, an estimate of the frequency of generalized tonic-clonic seizures, history of febrile convulsions, neurologic exam, intelligence, radiologic studies, and presumed etiology of the seizure disorder) was obtained from the hospital charts.

The only significant difference found between the 2 groups was that of an increased tendency for generalized tonic clonic seizures (defined as $>2 / y r)$ in the study group $(\mathrm{p}<.01)$.

We conclude that the presence of generalized spike wave discharges in patients with complex partial seizures and focal temporal epileptiform discharges may indicate a greater tendency towards generalized tonic-clonic seizures. The coexistence of this EEG phenomenon should not deter the clinician from further evaluation regarding surgery as the patient may still have a resectable lesion.

\section{1.}

\section{The Effects of Sacrificing Bridging Veins in Anterior Cor- pus Callostomy for Seizure Control}

R.C. CHAN, J.A. WADA and P.M. MOYES (Vancouver, British Columbia)

The authors report 14 patients who underwent anterior corpus callostomy for medical intractable seizures and bilateral epileptiform abnormalities on the EEG's. Excellent results were obtained in 13 patients. Follow-up ranges from 15 months to six years with a mean of 2.8 years. By converting generalized convulsions to focal seizure discharges, they can be controlled with moderate doses of anticonvulsive medications. Patients suffering from intractable seizures have a higher metabolic demand and increased vulnerability to venous infarction. Occlusion of small sagittal bridging veins prior to sectioning of the anterior corpus callosum may be disastrous. In nine patients one or more draining veins were divided with postoperative neurological deficits. The authors recommend the usage of osmotic agents and subarachnoid drains to decrease retraction ischemia, and turning a large frontoparietal craniotomy flap to improve visualization. The latter allows for better angulation of the operating microscope and offers higher opportunity to preserve the important venous structures. Postoperative generalized seizures were markedly reduced and were reported in only two patients who had sacrifice of their small bridging veins. Good control of focal seizures was accomplished in the group with bridging vein sacrifice, but a better result was obtained in those with an intact venous drainage system.

\section{2.}

\section{A Computerized EEG Data File Management System}

\section{J. PATRICK (Winnipeg, Manitoba)}

Current methods of storing and retrieving information pertaining to electroencephalograms (EEGs) are inadequate. We have therefore developed software to establish an on-line EEG Data File Management System for use with a microcomputer.

The software is written in BASIC, allowing the user and computer to communicate in "everyday" speech forms. Turnaround time is increased by directly entering data onto the computer and through responses to multiple choice statements. The system utilizes the terminology recommended by the International Federal of Societies for Electroencepha- lography and Clinical Neurophysiology. After completing an EEG recording the technologist enters data under three categories: 1) personal data (e.g. name, sex); 2) clinical-EEG recording data (e.g. cooperation, clinical states); 3) technical data (e.g. electrode placement, impedances). The physician's reporting format is structured to minimize intra- and inter-observer variability. Thus, EEG activities such as alpha, beta, theta are dealt with individually, and symmetry, frequency, amplitude, quantity, occurrence, reactivity, states in which the activity is seen in are all addressed. Billing and reports are automated, but interpretation can also be entered manually.

The system is cost effective and versatile, and will enable us to report, store and retrieve EEG data efficiently and accurately, thereby facilitating patient care and research.

113.

Markers of Altered Monoaminergic Function in Human Focal Epileptic Brain

R. BRIÈRE, A. SHERWIN, A. OLIVIER, Y. ROBITAILLE, F. QUESNEY and T. READER (Montréal, Québec)

Monoamines modulate cortical excitability and influence the threshold for epileptic discharge. Noradrenergic denervation facilitates focal spiking in experimental cobalt epilepsy and inhibitory effects also result from the action of certain drugs on central adrenoceptors. We recently observed a $58 \%$ inc rease in the activity of tyrosine hydroxylase (TH), the rate controlling enzyme in catecholamine synthesis, in spiking human epileptic cortex (Sherwin, A. et al., Neurology; in press). We now report adrenoceptor binding in paired samples from ECoG defined actively spiking areas and distal non-spiking cortex (control) excised from 11 patients undergoing neurosurgical therapy. Radioligands employed were ${ }^{3} \mathrm{H}$-Prazosin (PRZ; an alpha-I antagonist), and ${ }^{3} \mathrm{H}-\mathrm{Di}$ hydroalprenolol (DHA, a beta antagonist). Saturation curves ( 12 dilutions prepared from $150 \mathrm{mg}$ tissue samples from each site) were analyzed by Scatchard plots. Comparative binding parameters were: PRZ; spiking, Bmax $165.55 \pm 10.51 \mathrm{f} \mathrm{mol} / \mathrm{mg} P$; $\mathrm{Kd} 0.151 \pm 0.024 \mathrm{nM}$; nonspiking, Bmax 205.16 $\pm 17.12 ; \mathrm{Kd} 0.175 \pm 0.044$. $(\mathrm{p}<0.05$, paired $\mathrm{t}$ test, $\mathrm{n}=10$ ). DHA; spiking, $\mathrm{B} \max 131.40 \pm 25.68$; Kd 1.111 \pm 0.321 ; non-spiking, Bmax $34 \pm 5.90, \mathrm{Kd} 1.663 \pm 0.289(\mathrm{~N} . \mathrm{S}, \mathrm{N}=11)$.

The data show that spiking human epileptic cortex exhibits a relative increase in the activity of $\mathrm{TH}$ and a reduction in the number of alpha-1 postsynaptic receptor binding sites $(B \max )$ with no change in their affinity (Kd). Further study will be required to delineate further the role of central adrenoceptors in human focal epilepsy but this may lead to a new therapeutic approach.

114.

Seizure Disorder Associated Hydrocephalus

D. KEENE, E. VENTUREYRA, S. HAZIN, R. MIRELES and L. IVAN (Ottawa, Ontario)

It has been the impression of several neurosurgeons and pediatric neurologists that there is a higher frequency of seizure disorders amongst the hydrocephalic patients than the general population. A review of the literature failed to reveal any studies of this association. For that reason, a retrospective review of 218 hydrocephalic children attending the neurosurgical clinic at the Children's Hospital of Eastern Ontario was made. All patients had been followed for at least 2 years and had their surgery done at the Children's Hospital. It was noted that 75 patients (34\%) had seizures. This was higher than expected. An attempt to look at risk factors was made by comparing the seizure to the nonseizure group. The comparison included etiology of the hydrocephalus, type of hydrocephalus, number of shunt procedures, neurological and intellectual status and age of shunting procedure. Correlation between etiology of the hydrocephalus, the neurological status of the patient and occurrence of seizures was found. 
115. Myoclonic Twitching and Seizures Following Cardiopulmonary
Arrest

D.W. ZOCHODNE and G.B. YOUNG (London, Ontario)

Twenty-one patients had myoclonic jerks or seizures within 4 days after cardiac arrest. Thirteen males and 8 females ranged in age from 35 to 79 years. Myoclonic jerks were seen in 18, involving the head and neck in 8. Grand mal seizures occurred in isolation in 2 patients and in combination with myoclonic twitching in 4 patients. One had shivering episodes. In 16 patients an EEG was performed. Findings included suppression (6), burst-suppression (3), epileptiform activity (5) and other abnormalities. At $24-48$ hours post arrest all but 2 patients were comatose. Of these comatose patients 1 was discharged with major neurologic sequelae whereas the remainder (18) died in a vegetative state 1 to 18 days post arrest. Two patients discharged walking had preservation of arousal and brainstem reflexes at the time of assessment and EEG changes were mild. A third survivor with significant neurologic impairment had initially absent oculovestibular reflexes. His EEG showed theta coma. There were no survivors among patients with suppression, burst-suppression or epileptiform potentials on EEG.

The results indicate that seizures and myoclonic jerks frequently presage a poor neurologic outcome. Clinical and EEG findings may help clarify the prognosis.

116.

\section{The Syndrome of Focal Epilepsy and Bilateral Occipital Corti- cal Calcifications}

M. SAMMARITANO, F. ANDERMANN, A. GUBERMAN and D. MELANSON (Montreal, Quebec) (Ottawa, Ontario)

Three patients presenting with partial complex seizures were found to have extensive bilateral occipital cortical calcifications. They had no evidence for nevus flammeus or choroid angiomatosis and no explanation was found for the calcified lesions. Neurological examinations were normal, two patients were of normal intelligence and one was mildly mentally retarded. EEG's showed bitemporal independent epileptic foci in two patients, and a right posterior temporal and occipital focus in the third. Age at seizure onset was 11 yrs., 12 yrs. and "infancy", and cortical calcifications were first demonstrated at ages 14,43 and 6 yrs., respectively. CT scans showed bilateral occipital curvilinear symmetrical calcifications involving primarily the cortex. This syndrome has not been described previously. Only one patient with Sturge-Weber and bilateral intracranial calcifications has been reported; he also had atypical features such as leptomeningeal melanosis, angiomatosis of the skull and cerebral and cerebellar malformations. Unilateral cerebral changes of Sturge-Weber without nevus flammeus are rare but do occur. This syndrome is an unlikely diagnosis for our three patients with bilateral occipital calcifications in the absence of nevus flammeus. Bilateral cerebral calcifications in two patients with ALL in longterm remission subsequent to chemotherapy and radiotherapy have been reported and perinatal encephalitis is also known to cause such calcifications.

The cause of the calcifications in our patients is undetermined, and it is also uncertain whether clinical seizures originate from one or both hemispheres.

117.

\section{The Natural History Of The First Afebrile Seizure In Childhood}

J.M. DOOLEY, P. CAMFIELD, C. CAMFIELD, J. TIBBLES, B. GARNER and T. FUNG (Halifax, Nova Scotia)

The natural history of the first afebrile seizure in childhood is unknown. The EEG records in Nova Scotia are a valuable method of detecting first seizures, as $95 \%$ of physicians order an EEG following the first afebrile seizure. By reviewing the EEG reports between 1978-1982 we identified 168 children who had a first afebrile seizure followed by an EEG. Patients with an acute encephalopathy or with myoclonic, akinetic and absence seizures were excluded. Data was gathered by chart review and telephone contact with the parents. The overall rate of recurrence was $52 \%$, with $77 \%$ recurring within 1 year. The follow-up for those without recurrence was $31.4 \pm 16$ months.

Three factors (seizure type, focal epileptic discharges on EEG and abnormalities on neurological examination) increased the likelihood of recurrence. Recurrences occurred in $33 / 75$ with an initial generalized tonic-clonic seizure. This is significantly less than for those with complex partial $(15 / 19, \mathrm{p}<.02)$ or Sylvian seizures $(5 / 8)$ or focal with secondary generalization (28/55). Focal epileptic discharges on EEG were associated with a recurrence in 34/50 compared to 20/54 with a normal EEG ( $p=.01)$ Other EEG abnormalities were not predictive. Abnormalities on neurological examination increased the recurrence risk from $65 / 138$ to $22 / 30(p<.01)$. Anticonvulsant medication had little effect, with $62 / 115$ treated and $24 / 53$ untreated patients having further seizures.

We have shown that recurrences are common following a first afebrile seizure, especially with complex partial seizures. Anticonvulsants may not alter the risk of recurrence.

\section{8.}

\section{Technical Limitations of Prolonged Ambulatory EEG Recordings}

E. SHWEDYK, G. ARBEZ, J. SILL, S. SESIA. P. JAVAKAR and J.P. PATRICK (Winnipeg, Manitoba)

Prolonged ambulatory EEG (AEEG) cassette recording now have an established role in the diagnosis and investigation of epilepsy, particularly the detection of spike and wave phenomena. In this paper we present several technical aspects of a typical ambulatory system. Awareness of these technical aspects is important to obtain a reliable AEEG.

The system investigated consisted of a preamplifier unit as described by Ives, a 4 channel Oxford cassette recorder and an Oxford PB2 playback unit. Technical aspects important in any instrumentation system are those of noise level, amplitude dynamic range and frequency response. We found the noise level of Ives preamplifier to be of the order of $10 \mu \mathrm{V}$ peak. Thus low level EEG recordings $(<20 \mu \mathrm{V})$ should be interpreted with caution. The EEG signal amplitude's upper limit was determined by the cassette recorder head's saturation level which we measured to be in the range of $150-200 \mu \mathrm{V}$. The recording system has 3 time constants in the preamplifier, cassette recorder and playback unit which determines the system's low frequency response. The most crucial time constant setting was that of the playback unit. We have found that an improper setting (though still in the range of 0.1 to $0.3 \mathrm{sec}$ ) will lead to highly distorted spike and wave signals when these signals exceed the saturation level. This was confirmed both by direct observation and simulation.

In conclusion though AEEGs have a useful role in neurology a knowledge of the technical limitations is necessary to properly assess them.

The authors would like to acknowledge the support of the Children's Hospital of Winnipeg Research Foundation, Health and Welfare, Canada and NSERC.

\section{9.}

Spectral Analysis as a Method of Evaluation of Serial Electroencephalographic Changes in 45 Premature Infants

\section{E.A. MacDONALD, D.G. BRUNET and M.A. DiPAOLO (Kingston, Ontario)}

Forty-five premature infants have been recruited into a pilot study designed to determine whether computer analysis of background rhythms of the electroencephalogram improves the diagnostic accuracy of electroencephalography at this age. All infants were between 25-34 weeks gestational age at birth; this was established from maternal dates of last menstrual period and Dubowitz examination of the infant. Complete physical examinations and polygraphy were performed within 2 weeks 
of birth in most infants and repeated biweekly until the infant's discharge. On average, 3 recordings per infant were obtained.

Polygraphic recordings contained 12 channels of electroencephalogram, 2 channels of electro-oculogram, 1 channel of submental electromyogram and 1 channel of cardiopneumogram. The latter 4 channels were used to determine sleep state. Recordings lasted approximately one hour and behavioural observations were noted on the record. All records were stored on magnetic tape for computer analysis.

Preliminary results suggest that the graphic display of compressed spectral analysis (C.S.A.) shows major electroencephalographic abnormalities more readily than conventional records requiring visual interpretation. Quantitative measurements confirm the impression of previous investigators that the range of normal is wide.

120.

\section{NeonatalSeizures: Factors Predicting Response to Anticonvulsants}

\section{C.B. VAN ORMAN and H.Z. DARWISH (Calgary, Alberta)}

129 infants, representing $3.2 \%$ of all NICU admissions over a six year period, had seizures in the neonatal period. Infants who had metabolic or infectious etiologies for their seizures or who had not received an adequate loading dose (>15 mg/kg in the initial 24 hours) of either phenobarbital (PB) or phenytoin (PHT) were excluded from further analysis. Response to anticonvulsants was defined as cessation of seizure activity within 2 hours of completing the loading dose(s) of PB or PHT or both. 103 infants were reviewed of whom $82 \%$ received an adequate loading dose of PB initially $(22.3 \pm 6.3 \mathrm{mg} / \mathrm{kg})$. Only $32 \%$ of these infants responded to PB. With the addition of a loading dose of PHT $(18.5 \pm 2.8 \mathrm{mg} / \mathrm{kg})$ another $21 \%$ of infants stopped seizing. The response to $\mathrm{PB}$ alone was not improved among a subgroup receiving $25 \mathrm{mg} / \mathrm{kg}$ or more. A comparison of responders (R) to nonresponders (NR) showed no significant differences in gestational age $(R=37.1 \pm 5.4$, $\mathrm{NR}=34.6 \pm 5.6 \mathrm{wks})$, birth weight $(\mathrm{R}=2794 \pm 1041, \mathrm{NR}=2331 \pm$ $1119 \mathrm{gm}), 1 \mathrm{~min} .(\mathrm{R}=3.1 \pm 2.3, \mathrm{NR}=3.6 \pm 2.4)$ or $5 \mathrm{~min} .(\mathrm{R}=5.2$ $\pm 2.5, \mathrm{NR}=5.8 \pm 2.6)$ Apgar scores. The most common underlying neurologic disorders were hypoxic-ischemic encephalopathy $(\mathrm{R}=68 \%$, $\mathrm{NR}=56 \%)$, intracranial haemorrhage $(\mathrm{R}=26 \%, \mathrm{NR}=35 \%)$ and malformations $(\mathrm{R}=3 \%, \mathrm{NR}=9 \%)$. These differences were not statistically significant. $43 \%$ of all infants died: $60 \%$ of NR and $29 \%$ of R $(\mathrm{p}<0.01)$. As a retrospective study, comparisons between $\mathrm{PB}$ and PHT could not be made since the majority of infants $(82 \%)$ received PB first. Usually those who failed on PB received PHT in addition (43\% of study group). The response to PB was not dependent on the time of onset of seizures. Of those who failed PB therapy and whose onset of seizures was on the first day of life, response to added PHT was better than for those whose onset of seizures was after the first 24 hours $(p<0.1)$. Overall response toanticonvulsants was pooreramong infants with tonic seizures than for other seizure types $(p<0.02)$. This difference was accounted for by better response of other seizure types to a combination of $\mathrm{PB}+\mathrm{PHT}$ compared to a persistently poor response of tonic seizures to the combination $(p<0.05)$.

\section{1.}

\section{Prognosis of Childhood and Adolescent vs Adult Onset Multiple} Sclerosis

\section{T.J. MURRAY and J. SZERB (Halifax, Nova Scotia)}

At the Dalhousie Multiple Sclerosis Research Unit we studied 42 patients with the onset of definite multiple sclerosis before age 20 (average age at onset 15.8) and compared these to 400 cases of MS with onset between ages 20 and 40 (average age at onset 28.9 ), all with the disease longer than 10 years. Because of the recognized increased disability in patients with onset after age 40 , this latter group were not included in the comparison. In the younger onset group there were no differences in the rate of progression of disability, pattern of symptoms or clinical-anatomical involvement, or pattern of course progression. The younger group had a higher rate of epilepsy $(16.7 \%$ vs $4.2 \%)$, a younger age of measles ( 7.6 vs 9 years), and a longer duration between onset and diagnosis (8.1 vs 4.6 years). The younger group had more ocular and cerebellar involvement, but less pyramidal involvement.

In conclusion, although there are some differences in the pattern of involvement and features of childhood versus adult onset multiple sclerosis, the rate of progression and prognosis for disability is similar in both groups.

\section{2.}

\section{Non-MS Patients in an MS Unit}

\section{T.J. MURRAY and S.J. MURRAY (Halifax, Nova Scotia)}

Of the last 400 patients referred to the Dalhousie Multiple Sclerosis Research Unit 52 patients (13\%) were found not to have the disease. $52 \%$ of these patients had themselves raised the concern of multiple sclerosis to the referring physician. The most striking finding was that $46 \%$ of the group were medical personnel or involved with allied health groups. There were 9 nurses, 2 physicians, 2 medical receptionists, 1 pharmacist, and 3 multiple sclerosis society workers. 7 other patients were closely involved with the care of MS patients.

Assessment of the problems that led to the suspicion of multiple sclerosis showed that $28(52 \%)$ of the patients had a definable neurological disease other than multiple sclerosis, but more often the definable disorder was a benign syndrome. 14 (26\%) had a definable psychiatric disorder, such as acute or chronic anxiety, depression or hysteria. In the remaining 10 cases the patients had symptoms that were clearly not MS, but the cause was uncertain. The specific diagnosis and the basis for confusion with MS will be outlined.

123.

Risk Factors Associated with the Occurrence of Multiple Sclerosis (MS) in Individuals with/without an MS Family History

\section{S. WARREN, R. COCKERILL and K.G. WARREN (Edmonton, Alberta)}

It has been suggested that there may be several types of MS for which there are different risk factors. The purpose of this study was to compare risk factors among patients from families in which more than one case occurred to factors among patients with no evidence of familial predisposition. $157 \mathrm{MS}$ patients were matched to controls (friends and non-blood relatives) on age, sex, race, and residence in a high risk zone before age 15 . Both groups were questioned on pre-onset age occurrence of infectious diseases; urban/rural residence; animal exposure; diet; alcohol and tobacco use; personality; family background and illnesses. Two case-control subgroups were established for the analysis: one in which the cases had a family history of $M S(N=40)$ and one in which the cases did not $(N=107)$. Significantly more MS patients than controls in both subgroups reported: rural residence before age 15; eating non-inspected farm/game meat and drinking well water; a generally tense personality; injury within one year prior to onset age; crowding in childhood home; and a family history of diabetes. Only a few additional risk factors were associated with the disease among patients with no familial history. Significantly more MS patients without a family history than controls reported: rural residence immediately prior to onset age; a positive history of recurrent herpes labialis; and being smokers. There was little indication that risk factors differ among individuals with/without a family history of MS.

\section{4.}

\section{Myo-Inositol in Multiple Sclerosis}

G.B. YOUNG, W.J. HADER, K.G. WARREN, D.M. LOGAN and M. HISCOCK (London, Ontario) (Saskatoon, Saskatchewan)(Edmonton, Alberta) (Downsview, Ontario) (Saskatoon, Saskatchewan)

Pattern reversal evoked potential (PREP) testing was done in 4 trials in 9 patients with clinically definite multiple sclerosis (MS) and in 9 
age-sex matched healthy controls. Trial 1 was without medication; Trial 2 was after $500 \mathrm{mg}$. of oral myo-inositol (MI) daily for 2 weeks; Trial 3 followed $1000 \mathrm{mg}$. daily for 2 weeks. Then Trial 4 was after 2 weeks off medication.

Mean latencies of the $P_{100}$ wave were significantly greater in the MS group $(\mathrm{P}<.001)$ but did not alter during the 4 Trials. Mean amplitude and duration of the $P_{100}$ wave increased in Trial 2 and further in Trial 3 in the $M S$ but not in the control group $(P<.05)$. Mean amplitude and mean duration were identical for Trials 1 and 4 in both groups.

Using gas-liquid chromatography MI levels in cerebrospinal fluid (CSF) were determined in $55 \mathrm{MS}$ patients and in 20 controls. No significant differences were found.

We conclude that MS patients differ from healthy controls in their response to oral $\mathrm{MI}$ as measured by PREP testing. We could not show a deficiency of MI in CSF of MS patients, however.

\section{5.}

\section{Dichotic Paradigms in Multiple Sclerosis}

\section{T.J. MURRAY, J. JACOBSON and U. DEPPE (Halifax, Nova Scotia)}

Conventional audiological tests in multiple sclerosis are variable and usually unremarkable. More recently electrophysiological measurements such as the stapedial reflex and auditory brainstem evoked responses have shown more consistent and valuable abnormalities.

Because routine speech reception and discrimination scores have been unremarkable in MS patients, we wished to determine if newer, more specific distorted speech tests might provide further information.

20 definite MS patients with a history of brainstem symptoms were administered three commonly used dichotic speech paradigms. The tests included Consonant-Vowel (CV) Non Syllable test, the Synthetic Sentence Identification (SSI) test, and the Staggered Spondaic Word (SSW) test. Results of the test procedures were variable, with the higher percentage of abnormalities obtained with the CV test in which 11 out of 20 patients exceeded 2 S.D. An unexpected finding was a striking right ear advantage, with 16 or 20 patients exceeding the norm for the expected degree of lateralization.

These findings suggest that brainstem lesions may influence higher order auditory processing as measured by certain diachotic test procedures.

126.

Localization of the Dorsal Midbrain Syndrome by Nuclear Magnetic Resonance (NMR) in a Patient with Multiple Sclerosis (MS)

\section{A. COSTANTINO, S. BLACK. T. CARR and R.L. NICHOLSON (London, Ontario)}

A 45 year old woman presented with the acute onset of vertical diplopia sixteen years following an episode of isolated optic neuritis. Examination revealed optic atrophy (O.D.), upgaze paralysis with vertical nystagmus on attempted upward gaze, convergence-retraction nystagmus and convergence spasm. (These findings will be shown on a videotape.) The remainder of the neurological examination was normal. The laboratory finding of oligoclonal banding in her cerebrospinal fluid and the abnormal right visual evoked potential supported the diagnosis of clinically definite multiple sclerosis (MS). Prior to steroid treatment high-volume contrast-delayed computerized tomography using a GE 8800 scanner showed no definite lesions. Nuclear magnetic resonance (NMR) imaging with a 0.15 Tesla resistive magnet (Technicare) revealed bilateral periventricular abnormalities with areas of increased signal (prolonged T2) on spin echo sequences (time to echo $=120 \mathrm{msec}$.; repetition time $=1000 \mathrm{msec}$.). Such abnormalities have previously been described in patients with MS. Furthermore, a discrete area of prolonged $\mathrm{T} 2$ was seen in the dorsal midbrain on spin echo imaging in the sagittal plane. This finding provided a striking in vivo correlation of her dorsal midbrain syndrome, which, in isolation is an unusual clinical manifestation of MS. It illustrates the usefulness of this new imaging technique for the detection of relevant hemisphere and brainstem lesions in patients with MS.
127.

\section{The Importance of Recognizing Cardiac Disease in Benign} X-Linked Muscular Dystrophy

\author{
G.M. KLEIN, A.K.W. BROWNELL, D.G. WYSE and F.C. NATH \\ (Calgary, Alberta)
}

Emery-Dreifuss Muscular Dystrophy (EDMD) is a rare disease characterized by X-linked inheritance, the presence of contractures early in its course, an unusual pattern of muscle wasting and weakness and cardiac conduction abnormalities. Two patients with EDMD will be described. Although both had been diagnosed as having muscular dystrophy, neither was recognized as having the Emery-Dreifuss type. In one patient, aged 20 years, the diagnosis of EDMD was made at a time when he was hospitalized for a cardiac rhythm disturbance that required treatment with a permanent pacemaker. The second patient, aged 22 years, was subjected to cardiac studies after he was diagnosed as EDMD. Although asymptomatic, his cardiac studies revealed abnormalities requiring treatment with a permanent pacemaker. The importance of recognizing this subgroup of the benign X-linked muscular dystrophies is stressed because of the potentially fatal but treatable cardiac conduction abnormalities which always co-exist.

128.

IgG $\lambda$ Associated Neuropathy with Dramatic Response to Plasma Exchange and Steroid Therapy

J. JHAMANDAS, C. MELMED, D. FRAIL, I. DUNCAN, P. BRAUN and J. STEWART (Montreal, Quebec)

A 65 year old female developed a severe demyelinating neuropathy over 18 months. An $\mathrm{IgG}_{x}$ monoclonal gammopathy was discovered but there was no malignant plasma cell dyscrasia, amyloidosis or other known cause of neuropathy. Electrophysiological findings were consistent with severe demyelination and mild axonal involvement. Sural nerve biopsy showed a marked reduction in large myelinated fibres, evidence of demyelination and remyelination, and little axonal degeneration. Immunofluorescence studies showed $\lg G$ present around large fibres, in the perineurium and macrophages. Immunoperoxidase studies showed localization of IgG to myelin sheaths. Immunoelectroblot analysis of serum revealed IgM reacting with an unidentified myelin protein.

The patient was treated with corticosteroids and plasma exchange ( 16 treatments in 2 months) and made a dramatic recovery, becoming functionally normal in 3 months. On low dose corticosteroids she has remained well for 2 years, in spite of persistence of the IgG peak in the serum. This excellent response contrasts with the relatively poor results of similar therapy in patients with IgM associated neuropathy (Melmed et al., 1983). This variation in response may be due to differences in immuno-pathological mechanisms and differing degrees of axonal involvement.

129.

Thyrotoxic Hypokalemic Periodic Paralysis in a Teenaged Male

\section{B.J. PRIEUR and E.G. MURPHY (Toronto, Ontario)}

An 18 year-old male of Philippino origin presented with a 5 hour history of progressive paresthesias and weakness of the lower and upper limbs of such severity he was unable to sit or stand alone. This weakness gradually resolved over the following 24 hours. A history of 13 similar episodes over the preceding 18 month period was obtained, frequently precipitated by strenuous activity or following a large meal. There was no family history of similar episodes. Complaints of "weak shoulders", heat sensitivity, and a $4 \mathrm{lb}$. weight loss despite hyperphagia were also elicited. On examination, the patient was of slight build, with a resting heart rate of $100 / \mathrm{min}$., a fine rest tremor, and early lid sag. A diffusely enlarged, soft, non-tender thyroid gland was palpable. Motor examination revealed upper arm and shoulder girdle muscular atrophy. 
grade $4 / 3$ power in the upper extremities and grade $3 / 5$ power in the lower extremities, with diffuse areflexia. Admission serum $\mathrm{K}^{+}$was $1.7 \mathrm{mEq} / \mathrm{L}(\mathrm{N}=3.5-5.2)$. Serum thyroxine was $267 \mathrm{nmol} / \mathrm{L}(\mathrm{N}=50-155)$. CK was $471 \mathrm{U} / \mathrm{L}(\mathrm{N}<190)$. EMG was "myopathic" with polyphasic potentials in the deltoid and biceps muscles.

After placement on propylthiouracil $100 \mathrm{mg}$. three times daily he became clinically euthyroid. No further episodes of periodic paralysis have occurred during 8 months of follow-up. Muscle bulk, power, serum CK and EMG have returned to normal.

This patient's presentation of thyrotoxic hypokalemic periodic paralysis (THPP) is unusual with respect to his young age at the time of symptom onset. Approximately $8 \%$ of reported cases have developed paralytic attacks before age 19 years whereas $85 \%$ of cases present in the 3rd to 4th decades. Also unusual is the history of his episodic weakness appearing to pre-date the onset of thyrotoxicosis. In view of this patient's Philippino origin, it is interesting to note that the majority of case reports have occurred in people of Japanese racial background. As would be expected in THPP, the attacks of episodic weakness have resolved with treatment of the primary disorder.

\section{0.}

\section{Lateralized Diaphragmatic Palsy with Bulbar Lesions}

H.Z. DARWISH, D.M. COOPER and R.H.A. HASLAM (Calgary, Alberta)

We report two children who showed signs of acute unilateral weakness of diaphragmatic movements with medullary lesions. An 8 year old boy had a malignant brainstem glioma. Two weeks after acute onset of 6th nerve palsy and right hemiparesis, he had become quadriparetic, dysphonic, and showed left sternomastoid and tongue weakness. Pulmonary status deteriorated acutely in association with paradoxical inward movements of the right chest wall during inspiration. The chest $\mathrm{x}$-ray showed an elevated right diaphragmatic leaflet and the possibility of a phrenic nerve palsy was entertained. He was intubated and placed on PEEP. Improvement occurred with decadron and radiotherapy. A ? year old Chinese girl was admitted with sudden onset of right hemiplegia and nystagmus. Over 24 hours she deteriorated showing right sternomastoid weakness and right tongue weakness. She showed paradoxical movements of the left abdominal wall, and elevated left diaphragmatic leaflet on chest $x$-ray. Fluoroscopy confirmed left diaphragmatic palsy. She improved slowly over a period of 3 weeks. Neurodiagnostic studies, including selective angiography, did not demonstrate a specific lesion. She has improved spontaneously.

These cases suggest that upper motor neuron weakness of the diaphragm can mimic a phrenic nerve palsy. The diaphragmatic weakness is usually contralateral to the site of the lesion in the medulla, identified by cranial nerve X, XI, and XIl involvement. The reticulospinal tract input to the contralateral phrenic motor neuron pool may originate in the medulla at the level of cranial nerves $X$ and XII.

\section{1.}

The Polyneuropathy Associated with Critical Illness: Differentiation from Guillain-Barré Syndrome by Electrophysiological and Cerebrospinal Fluid Studies

\section{C.F. BOLTON, J.D. BROWN, A.F. HAHN, D. LAVERTY and W.} SIBBALD (London, Ontario)

We have observed a unique polyneuropathy occurring at the peak of critical illness (sepsis and multi-organ dysfunction). To determine if this polyneuropathy is simply a variant of Guillain-Barré syndrome (GBS), we compared 16 critically ill patients who had polyneuropathy and $18 \mathrm{GBS}$ patients (criteria: Asbury et al., 1978); both groups were investigated in the last six years by the same techniques and at similar stages of the polyneuropathy. Children and those GBS patients with a relapsing or progressing course were excluded.
The results in GBS patients were similar to those reported in the literature, and when compared to critically ill polyneuropathy, there was much less abnormal spontaneous activity in muscle, longer $M$ and $F$ wave latencies and a greater concentration of CSF protein. All comparisons were statistically significant. Electrophysiological follow-up at one month and six months was consistent with predominantly segmental remyelination in GBS and axonal regeneration in critically ill polyneuropathy.

We conclude that the polyneuropathy associated with critical illness and GBS are separate entities which can be differentiated in most patients by the above studies.

\section{2.}

Neurosarcoidosis Presenting as Reversible Dementia: Clinical Course and Management

\section{J.Y. CHU and T.A. GRAY (Toronto, Ontario)}

Three black male patients with neurosarcoidosis initially presented as progressive dementia with poor memory, psychomotor retardation and headache. The mean age of onset was 41.7 years with an average duration of symptoms of 6.7 months. The average follow-up was 3.1 years. CT scans of all three showed abnormal gyral enhancement with moderate ventricular dilatation while two had evidence of sarcoid involvement of the hypothalamic-pituitary area. CSF studies reviewed moderate lymphocytic pleocy tosis with elevated protein and depressed glucose. Non-caseating granulomas were found on liver biopsy of two patients while the third on lacrimal gland biopsy.

All three patients responded to oral prednisone with rapid improvement of their dementia but relapsed when dosage was reduced. One patient received 2,000 Rads. of cranial radiation in an attempt to reduce the maintenance dose of steroid but failed. His disease activity is currently being monitored by his CSF findings. Another patient required a V-P shunt for communicating hydrocephalus.

Neurosarcoidosis presenting as sarcoid meningitis is a rare cause of progressive dementia, however it is potentially reversible with steroid treatment. The clinical course, investigations and the use of CSF parameters to guide the dosage reduction of steroid will be discussed.

133.

\section{Tuberous Sclerosis and Wolff-Parkinson-White Syndrome}

\section{P.B. JAVAKAR and S.S. SESHIA (Winnipeg, Manitoba)}

Tuberous sclerosis (T.S.) is a neurocutaneous syndrome in which masses occur in various organs. Rhabdomyomas in the heart can cause arrhythmias. We report two children with T.S. and the Wolff-ParkinsonWhite (W.P.W.) syndrome. Case 1, R.P. developed supraventricular tachycardia (S.V.T.) at two weeks of age. An E.K.G. showed W.P.W. syndrome. He developed infantile spasms nine weeks later and was found to have white macules. C.T. scan showed intracranial calcification characteristic of T.S. Case 2, S.E., was admitted at three months of age with S.V.T. An E.K.G showed W.P.W. syndrome. He developed left and right focal motor seizures a fortnight later and a diagnosis of T.S. was considered although he had no skin lesions. A C.T. scan at 6 months of age was suggestive of T.S. White macules developed at one year and adenoma sebaceum at the age of 6 years.

Neurological symptoms are uncommon in patients with W.P.W. syndrome. Its association with T.S. in our case and in one reported previously suggests that the syndrome may be a manifestation of T.S. T.S. should be considered in children who have seizures and W.P.W. syndrome. Furthermore, EKGs to detect aberrations of conduction such as W.P.W. syndrome, may prove useful for family screening. 
134.

\section{Prolonged Neurologic Deficit Associated With Home Kerosene Heater Usage}

\section{C.E. MAXNER and L.P. HEFFERNAN (Halifax, Nova Scotia)}

Concerned about rising fuel expenses many North Americans reduced home ventilation and purchased portable kerosene space heaters purported to be both safe and efficient. Consumer interest groups originally suggested that kerosene heater safety should be reconsidered given the levels of harmful gases $\left(\mathrm{CO}, \mathrm{CO}_{2}, \mathrm{NO}_{2}, \mathrm{SO}_{2}\right)$ emitted. Interestingly very few case reports have appeared in the recent medical literature documenting harmful side-effects. We report the case of a married couple who simultaneously developed neurologic symptoms and signs - essentially consisting of mild encephalopathy with some focal features - associated with kerosene heater usage. One of the patients demonstrated a unilateral lenticular nucleus lucency on CT scan. Both patients display persistent mental status abnormalities documented by detailed neuropsychological testing at one year follow-up. The offending gaseous agent was presumed to be primarily carbon monoxide. We present this case to demonstrate an element of the spectrum of $\mathrm{CO}$ toxicity and as well to draw attention to the increasing data, including Consumer and Corporate Affairs Canada test results, suggesting potentially harmful levels of gaseous emissions from kerosene heaters if not used under proper ventilation conditions.

135.

Behavioral and Biochemical Correlates of Dopamine D1 and D2 Receptor Stimulation

\section{M.N. HASSAN, D. HIGGINS and S. FAHN (New York, New York)}

Dopamine (DA) agonists, therapeutically effective in Parkinson's disease, induce a variety of behavioral and biochemical effects in rodents. In order to correlate these effects with DA receptor subtypes, a series of DA agonists (apomorphine, bromocriptine, levodopa and two recently synthesised compounds - dihydroxy phenyl propylpyrolidine (DPPP) and SK\&F 38393-A) were investigated.

All agonists stimulated locomotor activity and reversed catalepsy in reserpinised rats, as well as elicited rotation in animals with unilateral lesions of the substantia nigra. All agonists, except SK\&F 38393-A induced stereotypy. Apomorphine and bromocriptine minimally, while SK\&F 38393-A and DPPP potently, stimulated striatal adenylate cyclase. All agonists, except SK\&F 38393-A inhibited both DA turnover and prolactin release.

Using the activation of striatal adenylate cyclase and the inhibition of prolactin release as indices of D1 and D2 receptor stimulation, respectively, it can be concluded that locomotor and rotational activities and catalepsy reversal are mediated by both D1 and D2 receptors, while stereotypy and the inhibition of DA turnover are mediated by D2 receptors. This study has indentified protocols which may be useful in screening new DA agonists for D1 and/or D2 properties.

136.

Neuropsychological and Electrophysiological Examination of a Patient with Wilson's Disease

\section{R.A. BORNSTEIN, D.R. McLEAN and K. HO (Edmonton, Alberta)}

Wilson's Disease is a rare disorder which has forms which preferentially involve the brain or liver. In the cerebral variant, the basal ganglia tend to be predominantly affected leading to prominent motor signs. In addition, the initial presentation may be with psychiatric symptoms. There are to date, no reports of formal neuropsychological evaluation of Wilson's Disease patients. We studied a 25 year old male patient approximately four months following symptom onset. Although motor functions were most severely affected, he also demonstrated impairment on a wide variety of cognitive, memory and intellectual tests. Auditory evoked potentials revealed a symmetric prolongation of the response in the III-V portion of the central conduction time suggesting a high mesencephalic lesion. The patient was treated with penicillamine and although he had a good physiological response in terms of copper excretion, his clinical state did not improve. This case may represent an unusually severe variant of Wilson's disease. In addition, the finding of diffuse cognitive impairment may be related to recent positron tomography studies which demonst rate globally decreased glucose metabolism in Wilson's disease.

137.

\section{An Unusual Case of Coma}

\section{HARVEY and R.G. PERRIN (Toronto, Ontario)}

A 32 year old previously healthy woman presented to a community hospital at 17:45 in deep coma. Her family stated she had felt unwell after lunch and had treated herself with antacids and Epsom salts. Before she lost consciousness, she complained of a feeling of intense heat. Intracranial catastrophe was suspected and she was transferred to our hospital.

When seen by Neurosurgical service at 22:00 she was in deep coma, breathing spontaneously, flaccid and areflexic. Computerized tomography of head and cerebrospinal fluid examination were unremarkable. Over next 12 hours and without specific therapy, patient recovered completely. Subsequently, lab. data returned indicating that at 04:00 her magnesium was $3.26 \mathrm{mmole} / 1$. These derangements were unsuspected.

Patient admitted to eating disorder and laxative abuse with consumption of up to $2 \mathrm{~kg}$. Epsom salts per week. We report this case because hypermagnesemia on basis of oral intake in healthy person has rarely been described.

138.

\section{Impaired $256 \mathrm{~Hz}$ Vibration in Parietal Lobe Disease}

\section{PRESTON, R. TSANG and G. BELANGER (Ottawa, Ontario)}

Two patients with markedly impaired 256 vibration in the hand were discovered to have contralateral inferior parietal lobe infarcts, involving supramarginal gyrus. The patients' studies which included serial neurological exams, CT scans, and sonatosensory evoked potential studies, will be presented. It is concluded that the supramarginal gyrus is likely important in the appreciation of $256 \mathrm{~Hz}$ vibration, and that impaired vibratory sensibility may have some localizing value in the clinical assessment of parietal lobe lesions.

139.

Domperidone in the Prevention of Gastrointestinal Symptoms Induced by Levodopa or Dopamine Agonist Therapy

J.D. GRIMES, A. KAPOOR, P. MAGNER, M. HASSAN and P. GRAY (Ottawa, Ontario)

Despite the use of dopa-decarboxylase inhibitors, about $10 \%$ of levodopa treated Parkinson patients have gastric symptoms. This incidence is higher with dopamine agonist therapy. Domperidone, a peripheral dopamine receptor blocker (with limited effect on central dopamine receptors) may help.

Ten Parkinson patients were enrolled in a double-blind trial divided into 2 consecutive 6 week periods; placebo or Domperidone $(20 \mathrm{mg}$ q.i.d.) and subsequently Domperidone. All ten patients had grade 2 or 3 nausea ( $0-3$ scale) and 6 patients had vomiting. Six patients were on Sinemet alone, 3 on bromocriptine and Sinemet, 1 on bromocriptine alone.

Two patients with severe vomiting could not complete the two week observation period on stable antiparkinsonian drug doses. In the doubleblind phase, 5 patients received placebo and 3 were on Domperidone. Three of the 5 placebo treated patients improved and all 3 of the Domperidone treated patients had complete clearing or significant 
improvement in gastric symptoms. Seven of the 8 patients in the open label phase had moderate or marked response to Domperidone. Gastric symptoms usually did not recur on late withdrawal of Domperidone.

Domperidone is effective for the short term control of nausea and vomiting in Parkinson patients treated with levodopa or dopamine agonists. Worsening of Parkinsonism does not appear to be a problem. The rate of placebo response and the frequent spontaneous clearing of gastric symptoms suggest that not all of the gastric upset in these patients is drug induced.

\section{0.}

The Management of De Novo Parkinson Disease With Combination Levodopa-Dopamine Agonist Therapy

\section{J.D. GRIMES and M.N. HASSAN (Ottawa, Ontario)}

Bromocriptine induces less late treatment complications than levodopa. Low dose levodopa is more effective than low dose bromocriptine and results in better initial response. There is now interest in early low dose combination levodopa-dopamine agonist therapy. Sixteen de novo patients have been treated.

Six patients were treated initially with levodopa-carbidopa and bromocriptine was added. Ten patients had levodopa-carbidopa added to bromocriptine (maximum $30 \mathrm{mg}$ daily). Levodopa-carbidopa dosages varied between 150 and $750 \mathrm{mg}$ daily (mean $300 \mathrm{mg}$ daily). The dose of bromocriptine varied from 7.5 to $30 \mathrm{mg}$ daily. The second drug was added at varying times between 8 months and 3 years.

Twelve of 16 patients demonstrated a clear initial synergistic effect of the drug combination. This was documented by clinical improvement and subsequent worsening with later withdrawal of bromocriptine. The clinical improvement with combination therapy was superior to that obtained with either drug alone.

Low dose combination levodopa-bromocriptine can give effective improvement in Parkinsonism. Because of the better initial response rate our preferred regime at present is to begin with levodopa-carbidopa and increase it to $300 \mathrm{mg}$ daily. Bromocriptine at a dose of 7.5 to $15 \mathrm{mg}$ is later added and may need to be increased to $30 \mathrm{mg}$ daily. The longest overall treatment time is 4 years and the longest time on combined therapy is now 2 years. To date none of these patients have developed fluctuations or dyskinesias. These results are encouraging, longer follow-up is necessary to assess this low dose combination therapy.

141.

CNS Toxoplasmosis Secondary to AIDS: A Hospital Experience With Two Cases

R.D. GROSCH and B.B. TESSLER (New Westminster, British Columbia)

A.M. - A 29 year old, white male, hemophiliac presented with a recent history of malaise, fatigue, night sweats, fever, and weight loss. A biopsy proven reactive lymphadenopathy occurred 3 years earlier. A headache, associated with confusion and focal CNS findings prompted a CT scan. Numerous enhancing lesions thought to be metastases were seen. The patient died shortly from uncal herniation. Post mortem study revealed generalized toxoplasmosis including the CNS (brain and spinal cord). Visual material (slides) will show CT scans, pertinent gross and microscopic pathology.

C.L. - A 58 year old male homosexual presented comatose following a recent history of weight loss and repeated respiratory infections. A generalized seizure occurred on admission. Pneumonia was seen on chest $x$-ray. Serial $C T$ scans showed clearing of multiple enhancing lesions after treatment with sulfadiazine and pyrimethamine. $T$ cell studies and anergy were compatible with AIDS. Patient's sexual partner has Kaposi's sarcoma of the tongue. Visual material (slides) will show CT scans, toxoplasmosis studies, pathology of Kaposi's sarcoma.

Summary slides or other visual material will briefly discuss AIDS, its neurological complications and treatment.

142.

Significance of Ultrastructurally Different Neurofibrillary Tangles (NFT) in Progressive Supranuclear Palsy (PSP)

R. F. NELSON, A. KAPOOR, V. MONTPETIT and A. GUBERMAN (Ottawa, Ontario)

In contrast to the ult rastructure of Alzheimer's NFT which has been well characterized as paired helical $10 \mathrm{~nm}$ filaments with $80 \mathrm{~nm}$ regular constrictions, the pathology of the neurofibrillary changes of PSP remains ill-defined. Until recently its fine structure was generally accepted as $15 \mathrm{~nm}$ straight filaments or tubules. Several authors have now described different electron microscopic findings. The aim of this paper is to document another atypical case and discuss the significance of these differences.

A 67 year old man was admitted for investigation of a prolonged neurological illness of 12 years' duration which started as hypersomnia after a 'flu-like' condition. His excessive sleepiness which lasted many years, was followed by falling spells and mental symptoms which eventually led to his admission to a nursing home. Examination shortly before his death revealed mild subcortical dementia, slight dysarthria, loss of convergence, upgave limitation, severely restricted saccadic eye movements with normal pursuits and normal tone of neck muscles. As well, he had reduced muscle bulk, prominent proximal fasciculations and a severely ataxic gait. EMG confirmed the denervation atrophy. He died of a ruptured abdominal aneurysm.

The histopathology was typical of PSP except for the presence of spinal motor neuron disease and minimal involvement of the dentate and red nuclei. Electron microscopy of NFT of midbrain median raphe disclosed $20-22 \mathrm{~nm}$ straight and twisted tubules with periodic $60-80 \mathrm{~nm}$ constrictions. The reason for this varied morphology of NFT in PSP is unclear. It is tempting to postulate that PSP is a syndrome or that neurofibrillary degeneration is a nonspecific reaction of the neuron to injury. Alternatively this varied ultrastructure might represent separate stages in the formation of the NFT. 